\title{
Application of topological quantum chemistry in electrides
}

\author{
Simin Nie, ${ }^{1,2, *}$ Yuting Qian, ${ }^{1,3, *}$ Jiacheng Gao, ${ }^{1,3}$ Zhong Fang, ${ }^{1,3}$ Hongming Weng, ${ }^{1,3,4, \dagger}$ and Zhijun Wang ${ }^{1,3, \ddagger}$ \\ ${ }^{1}$ Beijing National Laboratory for Condensed Matter Physics, \\ and Institute of Physics, Chinese Academy of Sciences, Beijing 100190, China \\ ${ }^{2}$ Department of Materials Science and Engineering, \\ Stanford University, Stanford, California 94305, USA \\ ${ }^{3}$ University of Chinese Academy of Sciences, Beijing 100049, China \\ ${ }^{4}$ Songshan Lake Materials Laboratory, Dongguan, Guangdong 523808, China
}

\begin{abstract}
The recently developed theory of topological quantum chemistry (TQC) has built a close connection between band representations in momentum space and orbital characters in real space. It provides an effective way to diagnose topological materials, leading to the discovery of lots of topological materials after the screening of all known nonmagnetic compounds. On the other hand, it can also efficiently reveal spacial orbital characters, including average charge centers and sitesymmetry characters. By using TQC theory with the computed irreducible representations in the first-principles calculations, we demonstrate that the electrides with excess electrons serving as anions at vacancies can be well identified by analyzing band representations (BRs), which cannot be expressed as a sum of atomic-orbital-induced band representations (aBRs). In fact, the floating bands (formed by the excess electrons) belong to the BRs induced from the "pseudo-orbitals" centered at vacancies. In other words, the electrides are proved to be unconventional ionic crystals, where a set of occupied bands is not a sum of aBRs but necessarily contains a BR from vacancies. The TQC theory provides a promising avenue to pursue more electride candidates in ionic crystals.
\end{abstract}

\section{INTRODUCTION}

Recently, the discovery of topological materials [1-10] has sprung up, and numerous nonmagnetic materials are predicted to be topologically nontrivial by first-principles calculations based on symmetry-based strategies, such as symmetry indicators [5, 6] and topological quantum chemistry (TQC) [7]. To be specific, the TQC theory first builds up the character tables for all $k$-points, and compatibility relations for all 230 space groups released on the Bilbao Crystalline Server [11], which make it possible to obtain the corresponding irreducible representations (irreps) of electronic states in the first-principles calculations (i.e., Irvsp [12]). For a given space group, a certain orbital ( $\rho$; labelled by the site-symmetry group) at sites $(q)$ can form a set of energy bands in momentum space (labelled by the irreps of $k$-points' little groups). This set of irreps at high-symmetry $k$-points is regraded as a BR of $\rho @ q$ in the TQC theory. If a BR $\rho @ q$ is a sum of other BRs, it is not elementary; otherwise, it is an elementary BR (eBR). Then, it constructs a complete list of (e)BRs, serving as its basic building blocks. By matching the irreps for a set of energy bands in a material with those of BRs, one can tell that these bands belong to a certain (e)BR $\rho @ q$. As thus, it makes a close link between the irreps in momentum space and the orbital characters in real space. The trivial [resp. topological (crystalline)] insulators satisfy compatibility relations and can [resp. cannot] be expressed as a sum of eBRs, while the enforced topological semimetals violate the compatibility

\footnotetext{
* These authors contributed equally to this work.

$\dagger$ hmweng@iphy.ac.cn

‡wzj@iphy.ac.cn
}

relations. Here, we emphasize that it not only can diagnose topology of energy bands in momentum space, but also can reveal orbital characters in real space (i.e., the average charge centers and site-symmetry characters) by doing the BR decomposition for a set of energy bands. It is also noted that for topologically trivial insulators, we can further define the unconventional ones, whose BR decomposition has to contain an essential BR induced from a "pseudo-orbital" centered at a vacancy, which can be characterized by real-space invariants [13]. In other words, the occupied bands of the unconventional insulators (also known as obstructed atomic limits [7, 14]) cannot be decomposed as a sum of aBRs (defined as the BR induced by the real atomic orbitals in crystals). To diagnose (topologically trivial) unconventional materials by doing the BR decomposition can be widely used in materials science [15-18], such as hydrogen storage materials, higher-order topological insulators (HOTIs), and electrides.

We here focus on the TQC application in the electrides. Electrides are defined as ionic crystals with excess electrons confined in particular vacancies [19-22], whose arrangement determines the properties of electrides and gives their classification by the dimensionality [23-26]. The anionic electrons, being not attached to any atom, bond or molecule, exhibit high electron mobility and low work function, which have been experimentally confirmed in $\mathrm{Ca}_{2} \mathrm{~N}$ [22]: a two dimensional (2D) electride with an excess electron per unit cell $\left(\left[\mathrm{Ca}_{2} \mathrm{~N}\right]^{+} \cdot e^{-}\right)$. The low work function of electrides is beneficial to induce band inversion, and to realize nontrivial band topology [18]. Recently, the concept of electrides with nontrivial band topology has attracted much attention for promising applications in quantum devices [18, 27-31]. Given the close relation between BRs and orbital characters, TQC may 
shed light on the origin of electrides in ionic materials.

Although there are many studies on searching for inorganic electrides, most of them are limited to compute charge distributions [23-26]. The symmetry analysis of electrides is lacking. In this work, the BR analysis of TQC theory provides a way to identify the origin of the bands around the Fermi level $\left(E_{F}\right)$ from their symmetry eigenvalues (or irreps) alone, which can be used to find new electride candidates effectively. We first introduce the concept of aBRs, which denote the BRs induced by real atomic orbitals in crystals. Then we demonstrate that the electrides are unconventional ionic crystals, whose occupied bands are decomposed as a sum of eBRs, but not a sum of aBRs. It is because that the BR decomposition necessarily contains a $\mathrm{BR}$ from vacancies (occupied by excess electrons). Lastly, some potential electrides are predicted in ionic crystals by the BR analysis of TQC theory, which can be further checked in future experiments.

\section{CALCULATION METHOD}

The Vienna ab initio simulation package (VASP) [32, 33 ] with the projector augmented wave method [34, 35] based on density functional theory was employed for the first-principles calculations. The generalized gradient approximation of Perdew-Burke-Ernzerhof type [36] was used for exchange-correlation potential. The cutoff energy for plane wave expansion was set to $500 \mathrm{eV}$. A $7 \times 7 \times 7$ Monkhorst-Pack grid for Brillouin zone sampling was supplied in the self-consistent process. A width of $0.02 \mathrm{eV}$ was adopted in the Gaussian smearing method for Fermi level determination. All the band structures were calculated without considering spin-orbit coupling. In order to get more reliable band structures for the $\mathrm{Ca}_{2} \mathrm{As}$ family, more accurate calculations with modified Becke-Johnson (mBJ) potential [37] were performed. The virtual crystal approximation [38] was employed to study the electronic structures of $\mathrm{K}_{2} \mathrm{O}_{0.5} \mathrm{~F}_{0.5}$ and $\mathrm{Sr}_{6} \mathrm{Ga}_{0.5} \mathrm{Ge}_{0.5} \mathrm{~N}_{5}$. The irreps are computed by the program Irvsp $[12,39]$, and the list of aBRs is given by the program pos2aBR [40] (see details in Supplementary Material [41]).

\section{RESULTS AND DISCUSSION}

To achieve an electride, it is empirically known that three criteria should be satisfied: excess electrons, lattice vacancies and suitable electronegativity of the elements. The previous search [23-26] for electrides have been done mainly by analyzing the charge density around $E_{F}$, where electron localization function analysis has proved to be effective. Here, by simply analyzing their symmetry eigenvalues (or irreps) alone at several maximal highsymmetry $k$-points in first-principles calculations, the BR analysis of TQC theory leads to the well understanding of three characteristics of electrides. First, the floating bands are induced from the BRs of vacancies, indicating that their average charge densities are located at the vacancies in real space. Second, due to the loose confinement, the floating bands are usually close to the $E_{F}$, which is beneficial to induce the band inversion and nontrivial band topology. Third, the excess anionic electrons in vacancies present a strong hydrogen affinity. The absorption of hydrogen usually moves those floating bands far below $E_{F}$ and lowers the total energy.

\section{A. Band representation of the pseudo-orbitals at voids}

We are interested in the compounds in the $\mathrm{Ca}_{2} \mathrm{As}$ family. The crystal $\mathrm{Ca}_{2} \mathrm{As}$ has a body-centered tetragonal structure and space group $14 / \mathrm{mmm}$ (\#139). The lattice parameters are $a=4.63 \AA, c=15.56 \AA$. The As and $\mathrm{Ca}(1)$ occupy the Wyckoff sites (WKS) $4 e(0,0, z)$ with $z=0.135$ and 0.328 , respectively, while $\mathrm{Ca}(2)$ is at the $4 c\left(0, \frac{1}{2}, 0\right)$, as shown in Fig. 1 and Table I. It is worth noting that the $2 \mathrm{~b}\left(0,0, \frac{1}{2}\right)$ positions denoted by blue-dashed circles are hollow. In the (001) planes, the $\mathrm{Ca}$ and As atoms form 2D square lattices of $\mathrm{Ca}$ and $\mathrm{As}$, respectively, which are stacked alternatively along the $z$ direction, as shown in Fig. 1(a).

The band structure of $\mathrm{Ca}_{2} \mathrm{As}$ with $\mathrm{mBJ}$ functional is shown in Fig. 1(b), where the size of the red and blue circles represents the weights of Ca- $d$ and As- $p$ orbitals, respectively. In addition to the presence of a global band

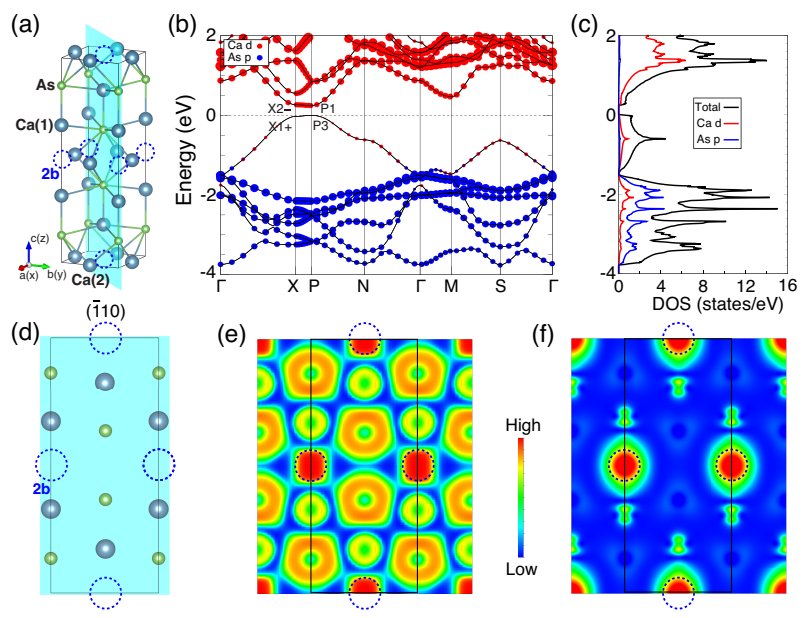

FIG. 1. (Color online) (a) Crystal structure and (d) (110) lattice plane of $\mathrm{Ca}_{2}$ As. (b) The mBJ band structure of $\mathrm{Ca}_{2}$ As with spectral weights of Ca- $d$ and As- $p$ orbitals represented by the size of the red and blue circles, respectively. (c) The orbital-resolved DOS of $\mathrm{Ca}_{2}$ As. (e) Electron localization function of the total electron density of $\mathrm{Ca}_{2} \mathrm{As}$, and (f) $\mathrm{PED}$ for the states in the energy range $-1.3 \mathrm{eV}$ to $0 \mathrm{eV}$ on the (110) plane of $\mathrm{Ca}_{2}$ As. Hereafter, the corresponding vacancy sites (i.e., WKS $2 b$ for $\mathrm{Ca}_{2} \mathrm{As}$ ) are depicted by blue-dashed circle. 
TABLE I. Atomic positions and aBRs of the compound $\mathrm{Ca}_{2} \mathrm{As}$.

\begin{tabular}{c|c|c|c|cc|c}
\hline \hline Atom & WKS $(q)$ & Site symm. & Conf. & Irreps $(\rho)$ & aBRs $(\rho @ q)$ \\
\hline As & $4 e$ & $4 m m$ & $4 p^{3}$ & $p_{z}$ & $: A_{1}$ & $A_{1} @ 4 e$ \\
& & & & $p_{x}, p_{y}$ & $: E$ & $E @ 4 e$ \\
\hline $\mathrm{Ca}(1)$ & $4 e$ & $4 m m$ & $4 s^{2}$ & $s$ & $: A_{1}$ & $A_{1} @ 4 e$ \\
\hline $\mathrm{Ca}(2)$ & $4 c$ & $m m m$ & $4 s^{2}$ & $s$ & $: A_{g}$ & $A_{g} @ 4 c$ \\
\hline \hline
\end{tabular}

TABLE II. Irreps and BRs for the 7 valence bands of $\mathrm{Ca}_{2} \mathrm{As}$. The BR colored in blue is generated by an excess electron $\mathrm{e}^{-}$ located at vacancies. Hereafter, the irreps are given in the order of increasing energy eigenvalues, and the number in the bracket denotes the degeneracy of the irrep.

\begin{tabular}{|c|c|c|c|c|c|}
\hline & $\Gamma(\mathrm{GM})$ & $\mathrm{M}$ & $\mathrm{P}$ & $\mathrm{X}$ & $\mathrm{N}$ \\
\hline \multirow{7}{*}{ Bands } & GM1+(1) & $\mathrm{M} 1+(1)$ & $\mathrm{P} 5(2)$ & $\mathrm{X} 4-(1)$ & $\mathrm{N} 2-(1)$ \\
\hline & $\mathrm{GM} 5+(2)$ & M3-(1) & P5(2) & X3-(1) & $\mathrm{N} 1+(1)$ \\
\hline & $\mathrm{GM} 1+(1)$ & $\mathrm{M} 5+(2)$ & P1(1) & $\mathrm{X} 4+(1)$ & $\mathrm{N} 1+(1)$ \\
\hline & GM3-(1) & M5-(2) & P3(1) & $\mathrm{X} 1+(1)$ & $\mathrm{N} 2+(1)$ \\
\hline & GM5-(2) & $\mathrm{M} 1+(1)$ & P3(1) & $\mathrm{X} 3+(1)$ & N2-(1) \\
\hline & & & & $\mathrm{X} 2-(1)$ & $\mathrm{N} 1-(1)$ \\
\hline & & & & $\mathrm{X} 1+(1)$ & N2-(1) \\
\hline \multicolumn{6}{|l|}{ BRs } \\
\hline \multirow[t]{2}{*}{$A_{1} @ 4 e$} & GM3-(1) & M3-(1) & P3(1) & $\mathrm{X} 2-(1)$ & $\mathrm{N} 2-(1)$ \\
\hline & $\mathrm{GM} 1+(1)$ & $\mathrm{M} 1+(1)$ & $\mathrm{P} 1(1)$ & $\mathrm{X} 1+(1)$ & $\mathrm{N} 1+(1)$ \\
\hline \multirow{4}{*}{$E @ 4 e$} & GM5-(2) & M5-(2) & $\mathrm{P} 5(2)$ & X3-(1) & $\mathrm{N} 1-(1)$ \\
\hline & GM5+(2) & $\mathrm{M} 5+(2)$ & P5(2) & $\mathrm{X} 3+(1)$ & $\mathrm{N} 1+(1)$ \\
\hline & & & & $\mathrm{X} 4-(1)$ & N2-(1) \\
\hline & & & & $\mathrm{X} 4+(1)$ & $\mathrm{N} 2+(1)$ \\
\hline$A_{1 g} @ 2 b$ & GM1+(1) & $\mathrm{M} 1+(1)$ & P3(1) & $\mathrm{X} 1+(1)$ & N2-(1) \\
\hline
\end{tabular}

gap in the band structure, one can also find that the valence bands are dominated by As- $p$ orbitals, while the conduction bands are mainly from $\mathrm{Ca}-d$ orbitals. Interestingly, since there are 7 valence bands but only 6 As- $p$ orbitals in a primitive cell, one can notice that the highest valence band in the energy range $-1.5 \mathrm{eV}$ to $0 \mathrm{eV}$ is not attributed to either As- $p$ or Ca- $d$ orbitals, which are consistent with the orbital-resolved density of states (DOS), as shown in Fig. 1(c). In what follows, we will show that the band does not belong to any aBR.

To check the orbital characters of the 7 well-isolated valence bands in the energy range $-4 \mathrm{eV}$ to $0 \mathrm{eV}$, we have computed the irreps for the high-symmetry $k$-point and done the analysis of BRs for these valence bands, which are presented in Table II. We find that these bands can be decomposed into a sum of BRs: $\left(A_{1}+E\right) @ 4 e \oplus A_{1 g} @ 2 b$. In view of all the aBRs of this compound given in Table I, the $\mathrm{BR}$ analysis indicates that the lowest six bands [i.e., $\left.\left(A_{1}+E\right) @ 4 e\right]$ are consistent with the states formed by three $p$-orbitals of As (i.e., WKS 4e), which agrees well with the fatted band structure [Fig. 1(b)]. Interestingly, the remaining band (i.e., $A_{1 g} @ 2 b$ ) is contributed from a specific eBR generated by a free electron $\mathrm{e}^{-}$located at the vacancy $2 b$. Therefore, the BR decomposition of $\mathrm{Ca}_{2} \mathrm{As}$ is not a sum of aBRs, suggesting that this inonic compound can be an electride candidate in terms of sym- metries and irreps.

The $A_{1 g} @ 2 b$ BR of excess electrons is further checked by the calculated charge density distribution. In Figs. 1(e) and 1(f), we plot the electron localization function of all valence states and partial electron density (PED) of the states in the energy range $-1.3 \mathrm{eV}$ to $0 \mathrm{eV}$ on (110) plane for $\mathrm{Ca}_{2} \mathrm{As}$, respectively, which both suggest the existence of excess electron distribution at the WKS $2 b$. Once the surface termination cuts through the vacancies (i.e., the charge centers of the floating bands), surface states would be expected $[18,42]$. The similar analysis can be also applied to other well-known electrides $\mathrm{Ca}_{2} \mathrm{~N}$ [22], $\mathrm{LaCl}$ [24], and $\mathrm{Ca}_{24} \mathrm{Al}_{28} \mathrm{O}_{64}$ (called C12A7 for short) [21] (see details in Sections B and C of the Supplementary Material[41]). Only using irreps at several high-symmetry $k$-points, the $\mathrm{BR}$ analysis of TQC theory tells that the blue bands belong to the BR from vacancies (i.e., $A_{1 g} @ 3 b$ for $\mathrm{Ca}_{2} \mathrm{~N}, A_{1 g} @ 3 b$ for $\mathrm{NaCl}$, and $A_{1} @ 2 b$ for C12A7 in Fig. 2), which are formed by excess electrons. It is confirmed by projecting the band structure onto the orbital states of the "empty atom" at the vacancy [22].

\section{B. Band inversion at the Fermi level}

It is well-known that topological materials usually have the band inversion near $E_{F}$. Compared with the states constrained by nuclei, the floating states are more conductive, and very close to the $E_{F}$. Given the very likely presence of the band inversion between the floating bands and other energy bands around $E_{F}$, it is natural to expect nontrivial band topology in electrides, resulting in the discovery of various topological states in electrides. The irrep and BR analysis will show that the band inversion in electrides is usually related to the BR of the vacancies.

Here, we take $\mathrm{Y}_{2} \mathrm{C}$ with as an example of topological electrides. It crystallizes in an anti- $\mathrm{CdCl}_{2}$ type structure with the space group of $R \overline{3} m$ (\#166). In the generalized gradient approximation calculation [36] [Fig. 3(a)], a band inversion is clearly denoted by the irreps (L2- and $\mathrm{L} 1+$ ) of the two low-energy bands at L, which contributes to a nodal-line structure traversing the full Brillouin zone without spin-orbit coupling [18]. The aBRs and the analysis of irreps for $\mathrm{Y}_{2} \mathrm{C}$ are shown in Table III and Table IV, respectively. After replacing the highest valence band irrep "L1+" by "?" (which denotes an arbitrary irrep), the only solution of BR decomposition for the valence bands

TABLE III. Atomic positions and aBRs of the compound $\mathrm{Y}_{2} \mathrm{C}$.

\begin{tabular}{c|c|c|c|cc|c}
\hline \hline Atom & WKS $(q)$ & Site symm. & Conf. & \multicolumn{2}{|c|}{ Irreps $(\rho)$} & $\operatorname{aBRs}(\rho @ q)$ \\
\hline $\mathrm{Y}$ & $6 c$ & $3 m$ & $5 s^{2} 5 d^{2}$ & $s / d$ & $: \rho$ & $\rho @ 6 c$ \\
\hline $\mathrm{C}$ & $3 a$ & $-3 m$ & $2 p^{2}$ & $p_{z}$ & $: A_{2 u}$ & $A_{2 u} @ 3 a$ \\
& & & & $p_{x}, p_{y}: E_{u}$ & $E_{u} @ 3 a$ \\
\hline \hline
\end{tabular}




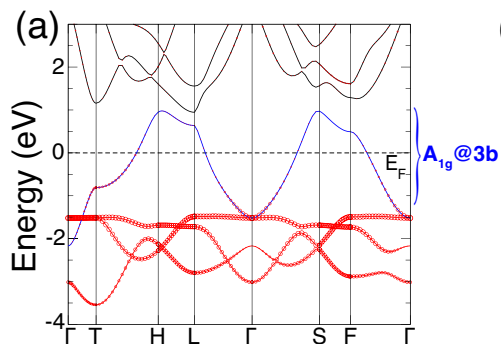

(d)

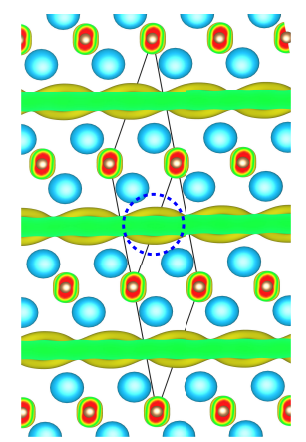

(b)

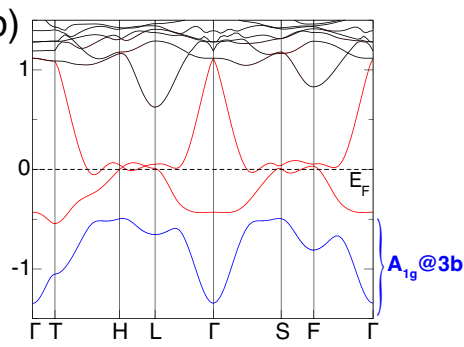

(e)

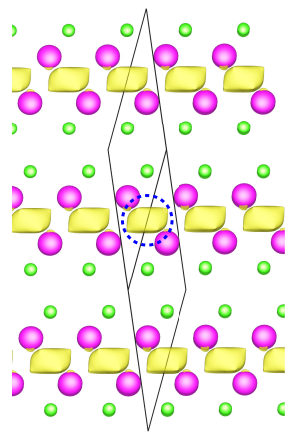

(c)

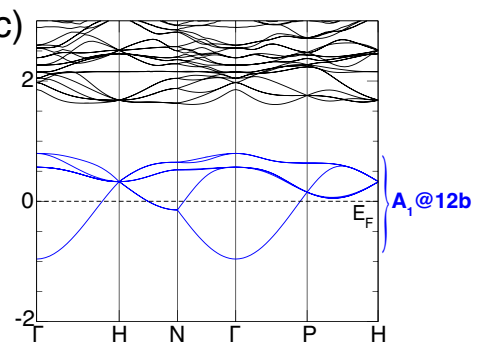

(f)

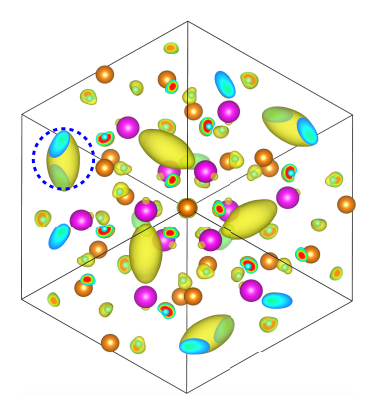

FIG. 2. (Color online) (a-c) The band structures of $\mathrm{Ca}_{2} \mathrm{~N}, \mathrm{LaCl}$, and $\mathrm{C} 12 \mathrm{~A} 7$, respectively. The spectral weight of $\mathrm{N} p$ orbitals for $\mathrm{Ca}_{2} \mathrm{~N}$ is denoted by the size of the red circles. (d-f) The calculated PED of the blue-colored bands for Ca $\mathrm{N}, \mathrm{LaCl}_{2}$, and $\mathrm{C} 12 \mathrm{~A} 7 \mathrm{in}$ the energy ranges of $[-1.5 \mathrm{eV}, 1 \mathrm{eV}],[-1.5 \mathrm{eV},-0.5 \mathrm{eV}]$ and $[-1 \mathrm{eV}, 1 \mathrm{eV}]$, respectively.

TABLE IV. Irreps and BRs (copied from TQC) for the four valence bands near the Fermi level of $\mathrm{Y}_{2} \mathrm{C}$. The question mark ("?") stands for any 1D irrep (i.e., the arbitrary irrep) at L. After analyzing the BRs of the occupied bands, one then find that the arbitrary irrep is solved to be L2-(1).

\begin{tabular}{c|c|c|c|c}
\hline \hline & $\Gamma$ & $\mathrm{T}$ & $\mathrm{F}$ & $\mathrm{L}$ \\
\hline \multirow{3}{*}{ Bands } & GM2-(1) & $\mathrm{T} 2-(1)$ & $\mathrm{F} 2-(1)$ & $\mathrm{L} 2-(1)$ \\
& GM1+(1) & $\mathrm{T} 3-(2)$ & $\mathrm{F} 2-(1)$ & $\mathrm{L} 2-(1)$ \\
& GM3-(2) & $\mathrm{T} 2-(1)$ & $\mathrm{F} 1-(1)$ & $\mathrm{L} 1-(1)$ \\
& & & $\mathrm{F} 1+(1)$ & $?(1)$ \\
\hline \hline BRs & \multicolumn{5}{|c}{} \\
\hline$A_{2 u} @ 3 a$ & GM2-(1) & $\mathrm{T} 2-(1)$ & $\mathrm{F} 2-(1)$ & $\mathrm{L} 2-(1)$ \\
\hline$E_{u} @ 3 a$ & GM3-(2) & $\mathrm{T} 3-(2)$ & $\mathrm{F} 1-(1) \oplus \mathrm{F} 2-(1)$ & $\mathrm{L} 1-(1) \oplus \mathrm{L} 2-(1)$ \\
\hline$A_{1 g} @ 3 b$ & GM1+(1) & $\mathrm{T} 2-(1)$ & $\mathrm{F} 1+(1)$ & $\mathrm{L} 2-(1)$ \\
\hline \hline
\end{tabular}

in $\mathrm{Y}_{2} \mathrm{C}$ is $\left(A_{2 u}+E_{u}\right) @ 3 a \oplus A_{1 g} @ 3 b$ with "? = L2-". The aBRs $\left(A_{2 u}+E_{u}\right) @ 3 a$ originate from C- $p$ orbitals, while the eBR $A_{1 g} @ 3 b$ is formed by an excess electron at the vacancy site $3 b$. The "?" is solved to be "L2-", suggesting that the $\mathrm{Y}_{2} \mathrm{C}$ has a band inversion at $\mathrm{L}$. The PED of the states colored in blue is also explored to confirm its electride feature, as shown in Fig. 3(b). The presence of charge distribution at the vacancies (i.e., WKS $3 b$ ) is consistent with the $\mathrm{BR}$ analysis of TQC theory.

The electrides $A_{2} B(A=\mathrm{Ca}, \mathrm{Sr}$, and $\mathrm{Ba} ; B=\mathrm{As}$, $\mathrm{Sb}$, and $\mathrm{Bi}$ ) with the space group of $I 4 / \mathrm{mmm}$ (\#139) are also reported topological nodal-line materials without spin-orbit coupling [30,43]. As aforementioned, $\mathrm{Ca}_{2} \mathrm{As}$ is a trivial electride with a global band gap. As the components vary from $\mathrm{Ca}(\mathrm{As})$, to $\mathrm{Sr}(\mathrm{Sb})$, to $\mathrm{Ba}(\mathrm{Bi})$ in the series of materials, the strength of the band inver- sion changes accordingly. In $\mathrm{Ba} 2 \mathrm{Bi}$, the band inversion [Fig. 3(c)] gives rise to a nodal line protected by the coexistence of space inversion and time-reversal symmetries. After replacing the highest valence band irreps X2- and $\mathrm{P} 1$ of $\mathrm{Ba}_{2} \mathrm{Bi}$ by "?", we can obtain the highest valence band from the eBR $A_{1 g} @ 2 b$ by solving the BR decomposition. Although the floating band of $A_{1 g} @ 2 b$ is not fully occupied and has a band inversion with the lowest conduction band, $\mathrm{Ba}_{2} \mathrm{Bi}$ can still be considered as an electride with nontrivial band topology. As expected, the PED of $\mathrm{Ba}_{2} \mathrm{Bi}$ shows the charge densities at the vacancy $2 b$ in Fig. 3(d). In fact, the $\mathrm{Ca}_{2} \mathrm{As}$ family have lots of compounds, which are all electride candidates (See mBJ band structures for all the compounds in Section D of the Supplementary Material[41]).

\section{Hydrogen absorption}

The electrides, with excess electrons serving as anions at vacancies, are very easy to absorb hydrogen atoms at the vacancies, which would move the floating bands far away from $E_{F}$. In order to understand the absorption process, we show the results of $\mathrm{Ca}_{5} \mathrm{P}_{3}$ and $\mathrm{Ba}_{3} \mathrm{CrN}_{3}$ as two paradigms. $\mathrm{Ca}_{5} \mathrm{P}_{3}$ crystallizes in a $\mathrm{Mn}_{5} \mathrm{Si}_{3}$-type structure with the space group of $\mathrm{P} 6_{3} / \mathrm{mcm}$ (\#193), and its electronic structure is shown in Fig. 4(a). The BR analysis shows that these blue-solid bands of $\mathrm{Ca}_{5} \mathrm{P}_{3}$ are the states formed by excess electrons at vacancies, since the eBR $A_{1 g} @ 2 b$ is determined by the obtained irreps labeled in Fig. 4(a). After absorption of hydrogen atoms at $2 b$ as shown in Fig. 4(c), the floating bands move far 

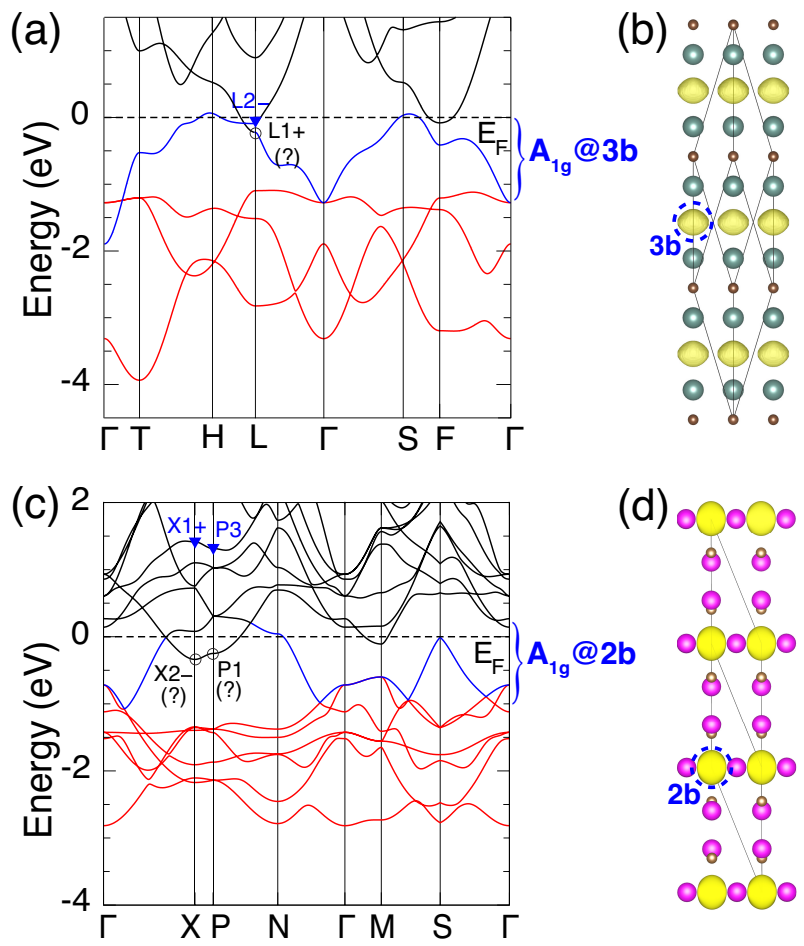

FIG. 3. (Color online) (a) Band structures of $\mathrm{Y}_{2} \mathrm{C}$ with generalized gradient approximation. (c) Band structures of $\mathrm{Ba}_{2} \mathrm{Bi}$ with mBJ modification. The irreps $\mathrm{L} 1+, \mathrm{X} 2-, \mathrm{P} 1$ replaced by "?" can be respectively assigned to the irrep L2-, X1+, P3 marked by blue filled triangles after solving the BR decomposition. The braces show the BRs produced by the bands in the corresponding energy ranges. The PED of the states for $\mathrm{Y}_{2} \mathrm{C}(\mathrm{b})$ and $\mathrm{Ba}_{2} \mathrm{Bi}(\mathrm{d})$ in the energy ranges $[-1.5 \mathrm{eV}, 0 \mathrm{eV}]$ and $[-1 \mathrm{eV}, 0.5 \mathrm{eV}]$, respectively.

below $E_{F}$ in $\mathrm{Ca}_{5} \mathrm{P}_{3} \mathrm{H}$ [Fig. 4(b)], because the hydrogen atoms will bond with the surrounding electrons producing a strong interaction, which is consistent with previous work [44]. The similar analysis of $\mathrm{Ba}_{3} \mathrm{CrN}_{3}$ can be found in Section E of the Supplementary Material[41]. Therefore, the process of adsorbing hydrogen atoms confirms the results of the BR analysis, and strongly supports the presence of the excess electrons at vacancies in electrides.

\section{Prediction of electride candidates}

Based on the above discussions on three aspects, we find that the BR analysis of TQC theory is very implementable in the understanding of various properties of electrides. We demonstrate that the electrides are unconventional ionic crystals, where a set of energy bands is not a sum of aBRs but necessarily contains a BR from vacancies. Guiding by this finding, we apply the BR analysis of TQC to predict some potential electrides in ionic crystals.

We first propose that $\mathrm{NaBaO}$ [45] with the structure of $\mathrm{P} 4 / \mathrm{nmm}$ (\#129) could be an electride with an ex- cess electron located at the vacancy $2 b$, as the BR of the floating bands [denoted by blue-colored lines and irreps in Fig. 4(d)] is elementary, and the average charge center is located at the vacancy. In fact, these floating bands are half-filled. By absorbing a hydrogen atom at $2 b$ [Fig. 4(f)], the floating bands move completely below $E_{F}$ [Fig. 4(e)].

Next we also find that $\mathrm{Sr}_{6} \mathrm{GaN}_{5}$ could be an electride after electron doping, i.e., $\mathrm{K}_{2} \mathrm{O}_{0.5} \mathrm{~F}_{0.5}$ and $\mathrm{Sr}_{6} \mathrm{Ga}_{0.5} \mathrm{Ge}_{0.5} \mathrm{~N}_{5}$. The band structure of $\mathrm{K}_{2} \mathrm{O}$ with space group Fm $\overline{3} m$ (\#225) [23] is shown in Fig. 5(a). Above $E_{F}$, we can see that there is an isolated band (bluecolored) in the energy range $1 \mathrm{eV}$ to $4 \mathrm{eV}$. With the obtained irreps at high symmetry $k$-points, the BR analysis shows that this band belongs to the eBR of $A_{1 g} @ 4 b$, which suggests that the band is formed by an excess electron at vacancy $4 b$. By replacing $50 \% \mathrm{O}$ with $\mathrm{F}$, the isolated band is partially occupied in $\mathrm{K}_{2} \mathrm{O}_{0.5} \mathrm{~F}_{0.5}$. The presence of a spherical charge distribution around the vacancy in the PED of this band [Fig. 5(b)] also suggests that $\mathrm{K}_{2} \mathrm{O}_{0.5} \mathrm{~F}_{0.5}$ is an electride. Heuristically, we propose that $\mathrm{Sr}_{6} \mathrm{GaN}_{5}$ with a hexagonal structure of $\mathrm{P}_{3} / \mathrm{mcm}$ (\#193) could also be an electride after electron doping. Its band structure is shown in Fig. 5(c). We find two well-isolated conduction bands (blue-colored) form the eBR of $A_{1 g} @ 2 b$, indicating the corresponding charge centers at $2 b$, which are consistent with the calculated PED [Fig. 5(d)]. By electron doping, the floating bands of $\mathrm{Sr}_{6} \mathrm{GaN}_{5}$ can be reached and become half-filled in $50 \%$ Ge-doped samples (i.e., $\mathrm{Sr}_{6} \mathrm{Ga}_{0.5} \mathrm{Ge}_{0.5} \mathrm{~N}_{5}$ ), as shown in Fig. 5(c). More details of these two predicted materials, and the results of another candidate (i.e., electron-doped $\mathrm{Sr}_{3} \mathrm{P}_{2}$ ) can be found in Section F of the Supplementary Material[41].

\section{E. Discussion}

Through the BR analysis of TQC with the obtained irreps at high-symmetry $k$-points, one can effectively identify the average electronic charge centers and the corresponding site-symmetry characters for a set of separated energy bands, providing an ideal avenue to both understand the essence of the well-known electrides and find new candidates in the future. Our methodology will facilitate the study of the interesting properties of electrides, such as high electron mobility and low work function. More interestingly, more fundamental physical phenomena can be found in the unconventional materials after considering interactions, such as $\mathrm{Sr}_{5} \mathrm{P}_{3}$ and $\mathrm{Zr}_{5} \mathrm{Sb}_{3}$.

The compound $\mathrm{Sr}_{5} \mathrm{P}_{3}$ has two different structures with the space group of $\mathrm{C} 2 / \mathrm{m}$ (\#12; quasi-hexagonal form) and the space group of $\mathrm{P} 6_{3} / \mathrm{mcm}$ (\#193; hexagonal form) at ambient and high pressures, respectively. The atoms in the quasi-hexagonal form deviate somewhat from that of the hexagonal form, and the hexagonal $\mathrm{Sr}_{5} \mathrm{P}_{3}$ shares the same crystal structure and similar band structure of $\mathrm{Ca}_{5} \mathrm{P}_{3}$. Thus, they are both expected to be elec- 

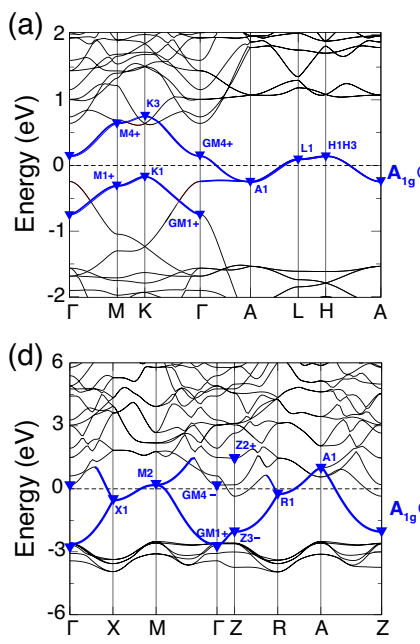

(b)

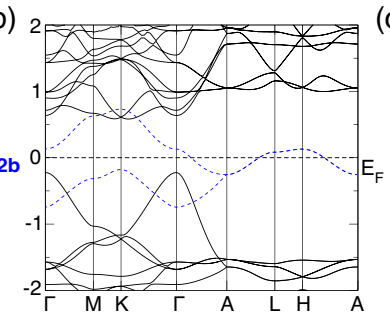

(e) 6

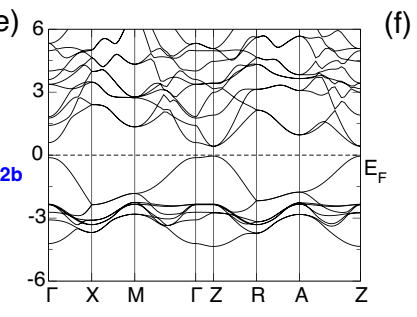

(c)

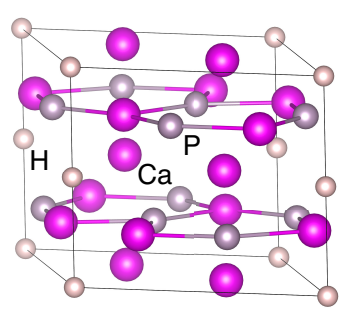

(f)

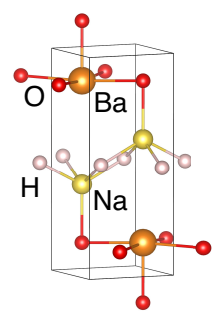

FIG. 4. (Color online) The band structures of $\mathrm{Ca}_{5} \mathrm{P}_{3}$ (a), $\mathrm{Ca}_{5} \mathrm{P}_{3} \mathrm{H}$ (b), $\mathrm{NaBaO}$ (d) and $\mathrm{NaBaOH}$ (e). The floating bands (blue-solid) in $\mathrm{Ca}_{5} \mathrm{P}_{3}$, and $\mathrm{NaBaO}$ denote electronic states of excess electrons, whose eBRs can be well determined by the obtained irreps. To facilitate the comparison, the irreps of particular bands are marked out, and the "disappeared" floating bands for $\mathrm{Ca}_{5} \mathrm{P}_{3} \mathrm{H}$ are depicted by blue-dashed lines in (b). (c,f) The crystal structures of $\mathrm{Ca}_{5} \mathrm{P}_{3} \mathrm{H}$ and $\mathrm{NaBaOH}$, respectively.

trides with half-filled floating bands. However, the obtained metallic band structure in the nonmagnetic calculations is in contrast with the observed insulator-type conductivity in experiments [26]. To remedy this discrepancy, the spin-polarized calculation and Coulomb-U effect have been considered in Ref. [26] and a tiny band gap
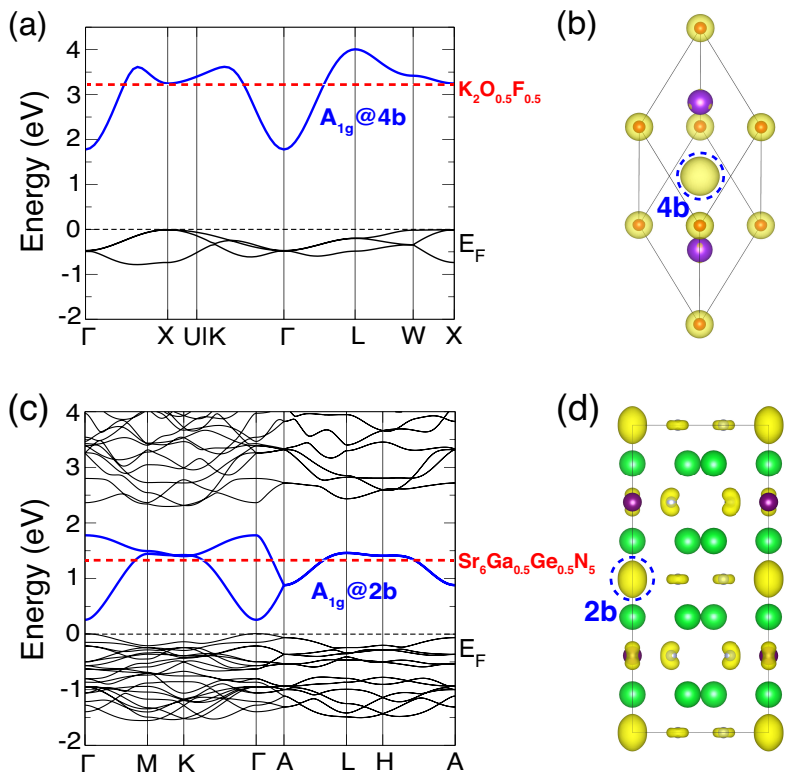

FIG. 5. (Color online) The calculated band structures and $\mathrm{PED}$ of the floating bands (blue-colored) for $\mathrm{K}_{2} \mathrm{O}(\mathrm{a}, \mathrm{b})$ and $\mathrm{Sr}_{6} \mathrm{GaN}_{5}$ (c,d). The PED of the states for $\mathrm{K}_{2} \mathrm{O}$ and $\mathrm{Sr}_{6} \mathrm{GaN}_{5}$ in the energy ranges $[1 \mathrm{eV}, 4 \mathrm{eV}]$ and $[0 \mathrm{eV}, 2 \mathrm{eV}]$, respectively. The Fermi levels of $\mathrm{K}_{2} \mathrm{O}_{0.5} \mathrm{~F}_{0.5}$ and $\mathrm{Sr}_{6} \mathrm{Ga}_{0.5} \mathrm{Ge}_{0.5} \mathrm{~N}_{5}$ are indicated by red-dashed lines, which are obtained by the virtual crystal approximation as implemented in the VASP. can be found, resulting in the formation of lower and upper Hubbard bands with DOS peaks below and above $\mathrm{E}_{F}$, respectively. They conjecture that this material may be a Mott insulator due to the effect of the on-site Coulomb interaction. In addition, the phase transition from the hexagonal form (high pressure) to the quasi-hexagonal form (ambient pressure) also suggests the presence of a commensurate charge-density-wave transition.

The compound $\mathrm{Zr}_{5} \mathrm{Sb}_{3}$ is experimentally found to be the first superconductor in the large family of compounds with $\mathrm{Mn}_{5} \mathrm{Si}_{3}$-type structure (\#193) [46], which are believed to be due to the electron-phonon coupling. Although the band structure is a little complicated, after carefully checking the irreps of the electronic states [15], we find that their irreps could be decomposed into a sum of BRs: $\left(A+B_{1}+B_{2}\right) @ 6 g$ (Sb atoms), $\left(A_{1}+E\right) @ 4 d$ [ $\operatorname{Zr}(1)$ atoms] and $A_{1}{ }^{\prime} @ 2 a$ (which is hollow). By electron counting, the floating bands of eBR $A_{1}{ }^{\prime} @ 2 a$ are $1 / 4$ filled, and create large Fermi surfaces. When the interstitial sites are filled by extra Sb atoms, the experimental measurements have also found that the superconductivity in $\mathrm{Zr}_{5} \mathrm{Sb}_{3+x}$ is suppressed. Similar results are also confirmed in $\mathrm{Zr}_{5} \mathrm{Sb}_{3} \mathrm{O}$ and $\mathrm{Zr}_{5} \mathrm{Sb}_{3} \mathrm{C}$. These experiments suggest that the superconductivity may be related to the floating electronic states.

\section{CONCLUSIONS}

In conclusion, we demonstrate that the analysis of irreps and BRs in the TQC theory provides an effective way to identify the origin of the energy bands around $\mathrm{E}_{F}$ from their symmetry eigenvalues (or irreps) alone, which is extremely useful to find new electride candidates. The electrides are proved to be unconventional ionic crystals, 
where a set of well-separated energy bands below/around the $E_{F}$ is not a sum of aBRs but necessarily contains a $\mathrm{BR}$ from vacancies. In addition, three characteristics of electrides can be well understood in the TQC theory. First, since there are floating bands with charge densities centered at vacancies in real space, surface states could emerge when the surface termination cuts through these vacancies. Second, as the excess electrons show the lack of strong confinement, low work function and floating bands close to $E_{F}$ are expected. Consequently, the band inversion and nontrivial band topology are very likely to happen in electrides. Third, the interstitial anionic electrons are easy to absorb hydrogen atoms, which would move the floating bands far away from $E_{F}$ and gain the benefit in total energy. More generally, the hydrogen storage materials and 2D HOTIs can be known as unconventional metal alloys and covalent compounds with trivial occupied bands being not a sum of aBRs, respectively. Anyway, the disagreement between the average charge centers and the atomic positions can be well diagnosed by the BR analysis of TQC theory, which can be widely used in these unconventional materials [15], such as electrides, hydrogen storage materials and HOTIs.

Acknowledgments - This work was supported by the National Natural Science Foundation of China (Grants No. 11974395), the Strategic Priority Research Program of Chinese Academy of Sciences (Grant No. XDB33000000), and the Center for Materials Genome. H.W. acknowledges support from the Ministry of Science and Technology of China under grant numbers 2016YFA0300600 and 2018YFA0305700, the Chinese Academy of Sciences under grant number XDB28000000, the Science Challenge Project (No. TZ2016004), the K. C. Wong Education Foundation (GJTD-2018-01), Beijing Municipal Science \& Technology Commission (Z181100004218001) and Beijing Natural Science Foundation (Z180008).
[1] F. Tang, H. C. Po, A. Vishwanath, and X. Wan, Nature 566, 486 (2019).

[2] F. Tang, H. C. Po, A. Vishwanath, and X. Wan, Nature Physics 15, 470 (2019).

[3] T. Zhang, Y. Jiang, Z. Song, H. Huang, Y. He, Z. Fang, H. Weng, and C. Fang, Nature 566, 475 (2019).

[4] M. G. Vergniory, L. Elcoro, C. Felser, N. Regnault, B. A. Bernevig, and Z. Wang, Nature 566, 480 (2019).

[5] H. C. Po, A. Vishwanath, and H. Watanabe, Nature communications 8, 1 (2017).

[6] Z. Song, T. Zhang, Z. Fang, and C. Fang, Nature communications 9, 1 (2018).

[7] B. Bradlyn, L. Elcoro, J. Cano, M. Vergniory, Z. Wang, C. Felser, M. I. Aroyo, and B. A. Bernevig, Nature 547, 298 (2017).

[8] T. H. Hsieh, H. Lin, J. Liu, W. Duan, A. Bansil, and L. Fu, Nature communications 3, 1 (2012).

[9] Z. Wang, A. Alexandradinata, R. J. Cava, and B. A. Bernevig, Nature 532, 189 (2016).

[10] J. Ma, C. Yi, B. Lv, Z. Wang, S. Nie, L. Wang, L. Kong, Y. Huang, P. Richard, P. Zhang, et al., Science advances 3, e1602415 (2017).

[11] "Bilbao crystallographic server," https://www.cryst. ehu.es/cgi-bin/cryst/programs/bandrep.pl.

[12] J. Gao, Q. Wu, C. Persson, and Z. Wang, Computer Physics Communications 261, 107760 (2021).

[13] Z.-D. Song, L. Elcoro, and B. A. Bernevig, Science 367, $794 \quad$ (2020), https://science.sciencemag.org/content/367/6479/794.full.pd

[14] J. Cano, B. Bradlyn, Z. Wang, L. Elcoro, M. Vergniory, C. Felser, M. Aroyo, and B. A. Bernevig, Physical Review B 97, 035139 (2018).

[15] Jiacheng Gao, Yuting Qian and Zhijun Wang. Work in progress.

[16] X.-L. Sheng, C. Chen, H. Liu, Z. Chen, Z.-M. Yu, Y. Zhao, and S. A. Yang, Physical Review Letters 123, 256402 (2019).

[17] E. Lee, R. Kim, J. Ahn, and B.-J. Yang, npj Quantum Materials 5, 1 (2020).
[18] M. Hirayama, S. Matsuishi, H. Hosono, and S. Murakami, Physical Review X 8, 031067 (2018).

[19] S. B. Dawes, D. L. Ward, R. H. Huang, and J. L. Dye, Journal of the American Chemical Society 108, 3534 (1986).

[20] J. L. Dye and M. G. DeBacker, Annual Review of Physical Chemistry 38, 271 (1987).

[21] S. Matsuishi, Y. Toda, M. Miyakawa, K. Hayashi, T. Kamiya, M. Hirano, I. Tanaka, and H. Hosono, Science 301, 626 (2003).

[22] K. Lee, S. W. Kim, Y. Toda, S. Matsuishi, and H. Hosono, Nature 494, 336 (2013).

[23] T. Tada, S. Takemoto, S. Matsuishi, and H. Hosono, Inorganic chemistry 53, 10347 (2014).

[24] Y. Zhang, H. Wang, Y. Wang, L. Zhang, and Y. Ma, Physical Review X 7, 011017 (2017).

[25] T. TADA, J. Wang, and H. Hosono, Journal of Computer Chemistry, Japan 16, 135 (2017).

[26] J. Wang, K. Hanzawa, H. Hiramatsu, J. Kim, N. Umezawa, K. Iwanaka, T. Tada, and H. Hosono, Journal of the American Chemical Society 139, 15668 (2017).

[27] H. Huang, K.-H. Jin, S. Zhang, and F. Liu, Nano letters 18, 1972 (2018).

[28] S. Nie, B. A. Bernevig, and Z. Wang, Phys. Rev. Research 3, L012028 (2021).

[29] S.-C. Zhu, L. Wang, J.-Y. Qu, J.-J. Wang, T. Frolov, X.-Q. Chen, and Q. Zhu, Physical Review Materials 3, 024205 (2019).

[30] X. Zhang, B. Fu, L. Jin, X. Dai, G. Liu, and Y. Yao, The Journal of Physical Chemistry C 123, 25871 (2019).

[31] S. Nie, H. Weng, and F. B. Prinz, Physical Review B 99, 035125 (2019).

[32] G. Kresse and J. Furthmüller, Phys. Rev. B 54, 11169 (1996).

[33] G. Kresse and J. Furthmüller, Computational Materials Science 6, 15 (1996).

[34] P. E. Blöchl, Phys. Rev. B 50, 17953 (1994).

[35] G. Kresse and D. Joubert, Phys. Rev. B 59, 1758 (1999). 
[36] J. P. Perdew, K. Burke, and M. Ernzerhof, Phys. Rev. Lett. 77, 3865 (1996).

[37] F. Tran and P. Blaha, Physical review letters 102, 226401 (2009).

[38] L. Nordheim, Ann. Phys 9, 607 (1931).

[39] https://github.com/zjwang11/irvsp/blob/master/ src_irvsp_v2.tar.gz.

[40] https://github.com/zjwang11/UnconvMat/blob/main/ src_pos2aBR.tar.gz.

[41] See Supplemental Material at http://link.aps.org/supplemental/xxx for the details on The code pos2aBR, Irrep and $\mathrm{BR}$ analysis of $\mathrm{Ca}_{2} \mathrm{~N}$ and $\mathrm{LaCl}$, Irrep and $\mathrm{BR}$ analysis of $\mathrm{C} 12 \mathrm{~A} 7$, The evolution of $\mathrm{mBJ}$ band structures of $\mathrm{A}_{2} \mathrm{~B}$, The absorption of hydrogen atoms in $\mathrm{Ba}_{3} \mathrm{CrN}_{3}$, The $\mathrm{BR}$ analysis of the predicted electrides.

[42] I. Tateishi, arXiv preprint arXiv:2004.02161 (2020).

[43] X. Zhang, Z.-M. Yu, Y. Lu, X.-L. Sheng, H. Y. Yang, and S. A. Yang, Physical Review B 97, 125143 (2018).

[44] L. S. Xie, L. M. Schoop, E. M. Seibel, Q. D. Gibson, W. Xie, and R. J. Cava, Apl Materials 3, 083602 (2015).

[45] J. Deng, J. Guo, and X. Chen, Journal of the American Chemical Society 142, 5234 (2020).

[46] B. Lv, X. Y. Zhu, B. Lorenz, F. Y. Wei, Y. Y. Xue, Z. P. Yin, G. Kotliar, and C. W. Chu, Phys. Rev. B 88, 134520 (2013).

[47] A. Togo and I. Tanaka, "Spglib: a software library for crystal symmetry search," (2018), arXiv:1808.01590 [cond-mat.mtrl-sci].

[48] B. Bradlyn, L. Elcoro, J. Cano, M. Vergniory, Z. Wang, C. Felser, M. Aroyo, and B. A. Bernevig, Nature 547, 298 (2017).

[49] M. Vergniory, L. Elcoro, Z. Wang, J. Cano, C. Felser, M. Aroyo, B. A. Bernevig, and B. Bradlyn, Physical Review E 96, 023310 (2017).

[50] J. Cano, B. Bradlyn, Z. Wang, L. Elcoro, M. Vergniory, C. Felser, M. Aroyo, and B. A. Bernevig, Physical review letters 120, 266401 (2018).

[51] M. I. Aroyo, J. Perez-Mato, D. Orobengoa, E. Tasci, G. de la Flor, and A. Kirov, Bulg. Chem. Commun 43, 183 (2011).

[52] M. I. Aroyo, J. M. Perez-Mato, C. Capillas, E. Kroumova, S. Ivantchev, G. Madariaga, A. Kirov, and H. Wondratschek, Zeitschrift für Kristallographie-Crystalline Materials 221, 15 (2006).

[53] M. I. Aroyo, A. Kirov, C. Capillas, J. Perez-Mato, and H. Wondratschek, Acta Crystallographica Section A: Foundations of Crystallography 62, 115 (2006).

[54] C. J. Bradley and A. P. Cracknell, "The mathematical theory of symmetry in solids." (1972).

[55] J. Wang, X. Sui, S. Gao, W. Duan, F. Liu, and B. Huang, Phys. Rev. Lett. 123, 206402 (2019).

[56] N. W. Falb, J. N. Neu, T. Besara, J. B. Whalen, D. J. Singh, and T. Siegrist, Inorganic chemistry 58, 3302 (2019). 


\section{SUPPLEMENTARY INFORMATION}

\section{A. The code pos $2 a B R$}

The program pos2aBR is developed to get the aBRs from a given crystal structure (i.e., POSCAR). The Spglib library [47] is needed for the program. After installing the Spglib library and copying libsymspg.a to the src_pos2aBR folder, the executable binary pos2aBR can be compiled by typing the following commands:

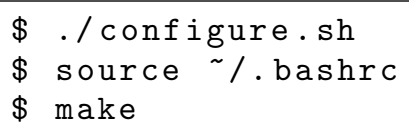

Before running pos2aBR, you would better standardize the POSCAR by using phonopy. A typical workflow of pos $2 \mathrm{aBR}$ is shown as follows:

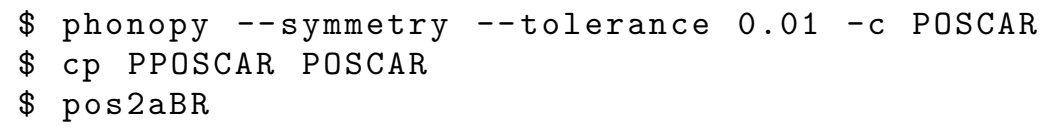

The program pos2aBR converts "POSCAR" to "POSCAR_std". One should use "POSCAR_std" to do the calculations within VASP, to be compatible with the program Irvsp and TQC work [14, 48-50]. In the meantime, its standard output contains the WKS $(q)$ for each atom and the irreps $(\rho)$ for atomic orbitals (i.e., $s, p, d)$. These BRs $(\rho @ q)$ are the aBRs for the crystal. An example of pos2aBR output for $\mathrm{Y}_{2} \mathrm{C}$ is shown in Fig. S1.

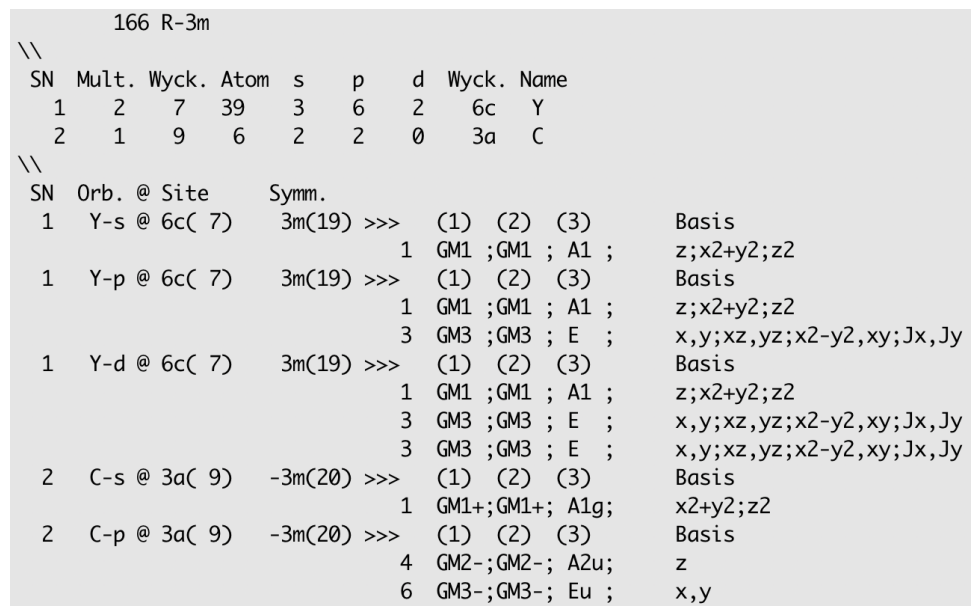

FIG. S1. The standard output of pos $2 a B R$ for $\mathrm{Y}_{2} \mathrm{C}$. The notations of point group irreps under "(1)" are used in Bilbao Crystallographic Server [51-53]. The notations under "(2)" and "(3)" follow Ref. [54]

\section{B. Irrep and $\mathrm{BR}$ analysis of $\mathrm{Ca}_{2} \mathrm{~N}$ and $\mathrm{LaCl}$}

$\mathrm{Ca}_{2} \mathrm{~N}$ and $\mathrm{LaCl}$ both crystallize in a hexagonal layered structure of space group $R \overline{3} m$ (\#166) with only three atoms (two calcium and one nitrogen atoms) and four atoms (two lanthanum and two chlorine atoms) in the primitive rhombohedral unit cells, respectively. The basic building blocks of $\mathrm{Ca}_{2} \mathrm{~N}$ and $\mathrm{LaCl}$ are tightly bound triple-layer and quadruple-layer structures, respectively. For the triple-layer structure in $\mathrm{Ca}_{2} \mathrm{~N}$, the nitrogen layer is sandwiched between two calcium layers in the sequence of $\mathrm{Ca}-\mathrm{N}-\mathrm{Ca}$, while two hexagonal lanthanum layers are sandwiched between two hexagonal chlorine layers in the sequence of Cl-La-La-Cl for the quadruple-layer structures. The adjacent triple layers and quadruple layers are stacked loosely, resulting in the floating electrons moving into the interlayer gaps [Fig. 2(d)] and intralayer gaps [Fig. 2(e)], respectively. Atomic positions and aBRs of $\mathrm{Ca}_{2} \mathrm{~N}$ and $\mathrm{LaCl}$ are shown in Table S1 and Table S3, respectively.

The band structure of $\mathrm{Ca}_{2} \mathrm{~N}$ shows three valence bands dominated by $\mathrm{N} p$ orbitals in the energy range $-4 \mathrm{eV}$ to -1.5 $\mathrm{eV}$, and a dispersive band traversing the Fermi level, as shown in Fig. 2(a). To understand the electride essence in 
terms of TQC theory, we calculate the irreps and BRs for the four bands near the Fermi level, as shown in Table S2. It is clear that the BR $A_{1 g} @ 3 b$ from these bands is an eBR, and generated by electrons located at the vacancy $3 b$, suggesting that $\mathrm{Ca}_{2} \mathrm{~N}$ is an electride.

The band structure of $\mathrm{LaCl}$ is shown in Fig. 2(b), and irreps and BRs for the three bands near the Fermi level of $\mathrm{LaCl}$ are shown in Table S4. Since there are band inversions at $\Gamma$ and $\mathrm{T}$, the irreps for the highest band at $\Gamma$ and $\mathrm{T}$ are unknown. After carefully analyzing the BRs, one can get that the unknown irreps should be GM1+(1) and T2-(1). Lastly, we find that these bands support an eBR $A_{1 g} @ 3 b$ generated by electrons located at the vacancy $3 b$, which suggests that $\mathrm{LaCl}$ is an electride.

TABLE S1. Atomic positions and aBRs of $\mathrm{Ca}_{2} \mathrm{~N}$ (space group $R \overline{3} m$ ).

\begin{tabular}{c|c|c|c|cc|c}
\hline \hline Atom & WKS $(q)$ & Site symm. & Conf. & \multicolumn{2}{|c|}{ Irreps $(\rho)$} & aBRs $(\rho @ q)$ \\
\hline Ca & $6 c$ & $3 m$ & $3 p^{6} 4 s^{2}$ & $s$ & $: A_{1}$ & $A_{1} @ 6 c$ \\
\cline { 4 - 6 } & & & & $p_{z}$ & $: A_{1}$ & $A_{1} @ 6 c$ \\
& & & & $p_{x}, p_{y}$ & $: E$ & $E @ 6 c$ \\
\hline $\mathrm{N}$ & $3 a$ & $-3 m$ & $2 s^{2} 2 p^{3}$ & $s$ & $: A_{1 g}$ & $A_{1 g} @ 3 a$ \\
\cline { 4 - 6 } & & & & $p_{z}$ & $: A_{2 u}$ & $A_{2 u} @ 3 a$ \\
& & & $p_{x}, p_{y}$ & $: E_{u}$ & $E_{u} @ 3 a$ \\
\hline \hline
\end{tabular}

TABLE S2. Irreps and BRs for the four bands near the Fermi level of $\mathrm{Ca}_{2} \mathrm{~N}$.

\begin{tabular}{c|c|c|c|c}
\hline \hline & $\Gamma$ & $\mathrm{T}$ & $\mathrm{F}$ & $\mathrm{L}$ \\
\hline & GM1+(1) & $\mathrm{T} 2-(1)$ & $\mathrm{F} 2-(1)$ & $\mathrm{L} 2-(1)$ \\
Bands & GM2-(1) & $\mathrm{T} 2-(1)$ & $\mathrm{F} 2-(1)$ & $\mathrm{L} 2-(1)$ \\
& GM3-(2) & $\mathrm{T} 3-(2)$ & $\mathrm{F} 1-(1)$ & $\mathrm{L} 1-(1)$ \\
& & & $\mathrm{F} 1+(1)$ & $\mathrm{L} 2-(1)$ \\
\hline \hline BRs & \multicolumn{3}{|c}{} \\
\hline$A_{2 u} @ 3 a$ & GM2-(1) & $\mathrm{T} 2-(1)$ & $\mathrm{F} 2-(1)$ & $\mathrm{L} 2-(1)$ \\
\hline$E_{u} @ 3 a$ & GM3-(2) & $\mathrm{T} 3-(2)$ & $\mathrm{F} 1-(1) \oplus \mathrm{F} 2-(1)$ & $\mathrm{L} 1-(1) \oplus \mathrm{L} 2-(1)$ \\
\hline$A_{1 g} @ 3 b$ & GM1+(1) & $\mathrm{T} 2-(1)$ & $\mathrm{F} 1+(1)$ & $\mathrm{L} 2-(1)$ \\
\hline \hline
\end{tabular}

\section{Irrep and BR analysis of C12A7}

C12A7 with low work function (about $0.6 \mathrm{eV}$ ) is a room-temperature electride, whose unusual properties are due to its special crystalline structure with the space group of $I \overline{4} 3 d(\# 220)$. The stoichiometric cubic unit cell is represented by the formula $\left[\mathrm{Ca}_{24} \mathrm{Al}_{28} \mathrm{O}_{64}\right]^{4+}$. $\left[\mathrm{O}^{2-}\right]_{2}$. The first term $\left[\mathrm{Ca}_{24} \mathrm{Al}_{28} \mathrm{O}_{64}\right]^{4+}$ denotes a positively charged framework built of twelve cages, while the second term $\left[\mathrm{O}^{2-}\right]_{2}$ represents two extra-framework oxygen ions occupying two of the twelve cages. By reduction treatment, the extra-framework oxygen ions can be replaced with electrons acting as anions, leading to the formation of the electride C12A7. Atomic positions and aBRs of C12A7 are shown in Table S5.

The band structure of C12A7 is shown in Fig. 2(c), irreps and BRs for the six bands near the Fermi level of C12A7 are shown in Table S6. This six bands support an eBR $A_{1} @ 12 b$ located at the vacancy $12 b$, as shown in Fig. 2(f), which confirms that $\mathrm{C} 12 \mathrm{~A} 7$ is an electride.

\section{The evolution of mBJ band structures of $A_{2} B$}

$A_{2} B(A=\mathrm{Ca}$, $\mathrm{Sr}$, and $\mathrm{Ba} ; B=\mathrm{As}, \mathrm{Sb}$, and $\mathrm{Bi})$ is a large family of isostructural materials hosting similar electronic structures. They are known as electrides before, and recently draw intensive research interest due to the presence of nontrivial band topology. In order to better understand the relation between these two different properties, we calculate the mBJ band structures of $A_{2} B$, as shown in Fig. S2. Although increasing the weights of the pnicogen elements doesn't change the band structure too much, the heavier alkaline earth metal in $A_{2} B$ leads to the larger band inversion. 
TABLE S3. Atomic positions and aBRs of $\mathrm{LaCl}$ (space group $R \overline{3} m$ ).

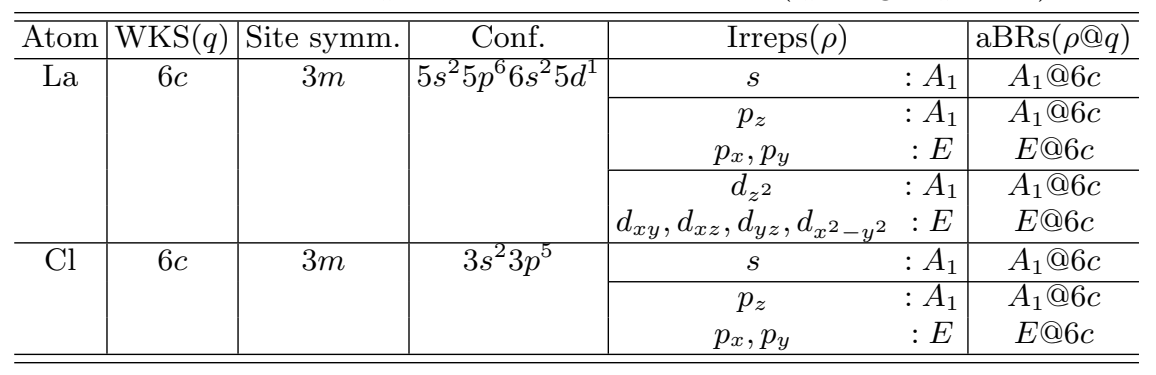

TABLE S4. Irreps and BRs for the three bands near the Fermi level of LaCl. The question mark ("?") stands for any one-dimensional (1D) irrep (i.e., the unknown irrep) at $\Gamma$ and $T$. After analyzing the BRs, one then can conjecture that the unknown irrep should be GM1+(1) and T2-(1).

\begin{tabular}{c|c|c|c|c}
\hline \hline & $\Gamma$ & $\mathrm{T}$ & $\mathrm{F}$ & $\mathrm{L}$ \\
\hline Bands & GM1+(1) & $\mathrm{T} 2-(1)$ & $\mathrm{F} 1+(1)$ & $\mathrm{L} 2-(1)$ \\
& GM2-(1) & $\mathrm{T} 1+(1)$ & $\mathrm{F} 1+(1)$ & $\mathrm{L} 1+(1)$ \\
& $?(1)$ & $?(1)$ & $\mathrm{F} 2-(1)$ & $\mathrm{L} 2-(1)$ \\
\hline \hline BRs (one of cases) & \multicolumn{4}{|l}{} \\
\hline$A_{1 g} @ 3 a$ & GM1+(1) & $\mathrm{T} 1+(1)$ & $\mathrm{F} 1+(1)$ & $\mathrm{L} 1+(1)$ \\
\hline$A_{2 u} @ 3 a$ & GM2-(1) & $\mathrm{T} 2-(1)$ & $\mathrm{F} 2-(1)$ & $\mathrm{L} 2-(1)$ \\
\hline$A_{1 g} @ 3 b$ & GM1+(1) & $\mathrm{T} 2-(1)$ & $\mathrm{F} 1+(1)$ & $\mathrm{L} 2-(1)$ \\
\hline \hline
\end{tabular}

TABLE S5. Atomic positions and aBRs of C12A7 (space group $I \overline{4} 3 d$ ).

\begin{tabular}{c|c|c|c|cc|c}
\hline \hline Atom & WKS $(q)$ & Site symm. & Conf. & \multicolumn{2}{|c|}{ Irreps $(\rho)$} & aBRs $(\rho @ q)$ \\
\hline $\mathrm{Ca}$ & $24 d$ & 2 & $3 p^{6} 4 s^{2}$ & $s$ & $: A$ & $A @ 24 d$ \\
\cline { 5 - 7 } & & & & $p_{z}$ & $: A$ & $A @ 24 d$ \\
& & & & $p_{x}, p_{y}$ & $: B$ & $B @ 24 d$ \\
\hline $\mathrm{Al}(1)$ & $12 a$ & -4 & $3 s^{2} 3 p^{1}$ & $s$ & $: A$ & $A @ 12 a$ \\
\cline { 4 - 6 } & & & & $p_{z}$ & $: B$ & $B @ 12 a$ \\
& & & & $p_{x}$ & $:{ }^{2} E$ & ${ }^{2} E @ 12 a$ \\
& & & & $p_{y}$ & $:{ }^{1} E$ & ${ }^{1} E @ 12 a$ \\
\hline $\mathrm{Al}(2)$ & $16 c$ & 3 & $3 s^{2} 3 p^{1}$ & $s$ & $: A_{1}$ & $A_{1} @ 16 c$ \\
\cline { 4 - 6 } & & & & $p_{z}$ & $: A_{1}$ & $A_{1} @ 16 c$ \\
& & & & $p_{y}$ & $:{ }^{2} E$ & ${ }^{2} E @ 16 c$ \\
& & & & $p_{x}$ & $:{ }^{1} E$ & ${ }^{1} E @ 16 c$ \\
\hline $\mathrm{O}(1)$ & $16 c$ & 3 & $2 s^{2} 2 p^{4}$ & $s$ & $: A_{1}$ & $A_{1} @ 16 c$ \\
\cline { 4 - 6 } & & & & $p_{z}$ & $: A_{1}$ & $A_{1} @ 16 c$ \\
& & & & $p_{y}$ & $:{ }^{2} E$ & ${ }^{2} E @ 16 c$ \\
& & & & $p_{x}$ & $:{ }^{1} E$ & ${ }^{1} E @ 16 c$ \\
\hline $\mathrm{O}(2)$ & $48 e$ & 1 & $2 s^{2} 2 p^{4}$ & $s$ & $: A$ & $A @ 48 e$ \\
\cline { 4 - 7 } & & & & $p_{z}, p_{x}, p_{y}$ & $: A$ & $A @ 48 e$ \\
\hline \hline
\end{tabular}

TABLE S6. Irreps and BRs for the six bands near the Fermi level of C12A7.

\begin{tabular}{c|c|c|c|c}
\hline \hline & $\Gamma$ & $\mathrm{H}$ & $\mathrm{P}$ & $\mathrm{N}$ \\
\hline & GM1(1) & $\mathrm{H} 4 \mathrm{H} 5(6)$ & $\mathrm{P} 3(4)$ & $\mathrm{N} 1(2)$ \\
Bands & GM5(3) & & $\mathrm{P} 2(2)$ & $\mathrm{N} 1(2)$ \\
& GM3(2) & & $\mathrm{N} 1(2)$ \\
\hline \hline BRs & \multicolumn{3}{|l}{} \\
\hline$A_{1} @ 12 b$ & $\mathrm{GM} 1(1) \oplus \mathrm{GM} 5(3) \oplus \mathrm{GM} 3(2)$ & $\mathrm{H} 4 \mathrm{H} 5(6)$ & $\mathrm{P} 3(4) \oplus \mathrm{P} 2(2)$ & $3 \mathrm{~N} 1(2)$ \\
\hline \hline
\end{tabular}



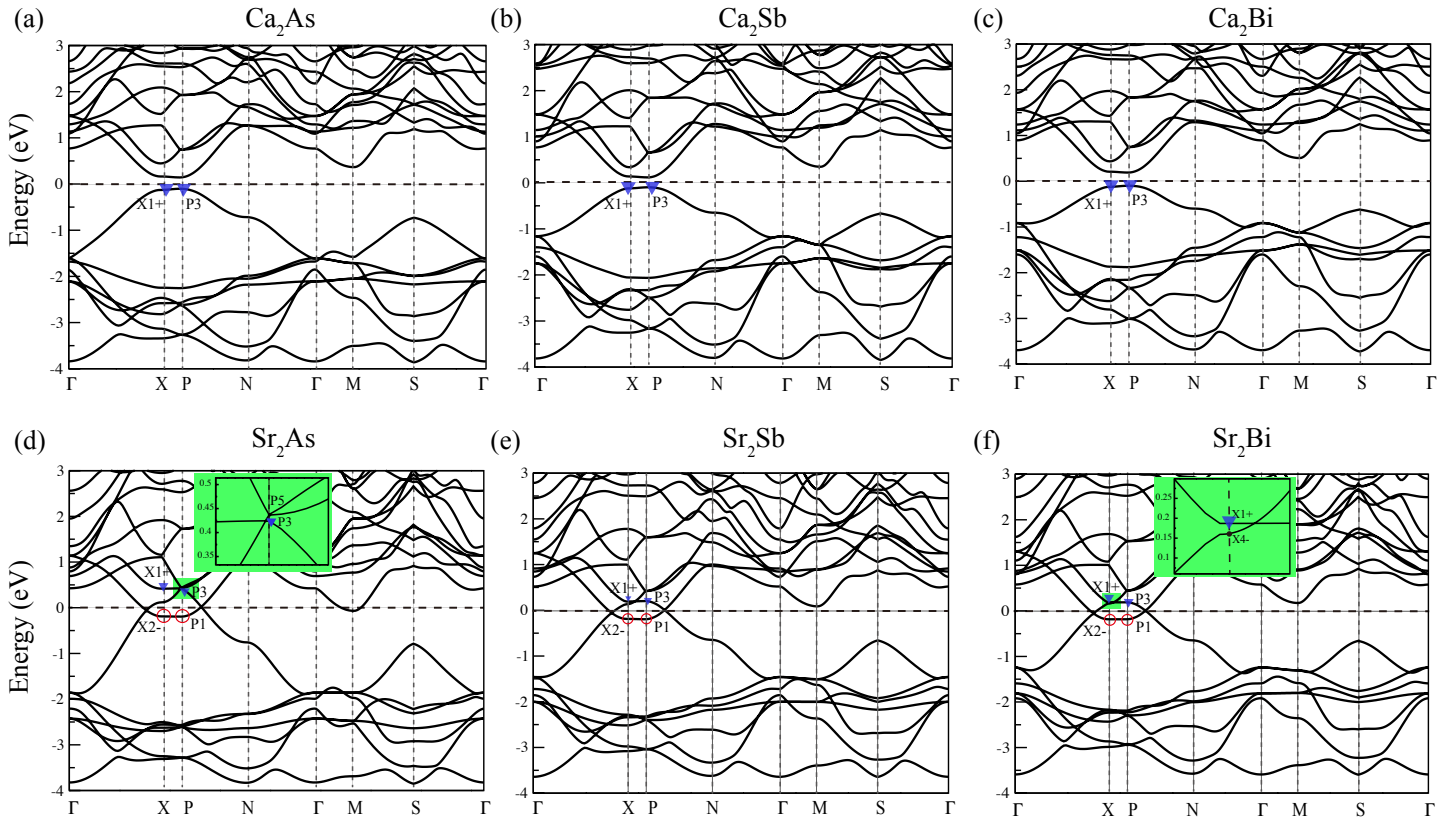

(e)

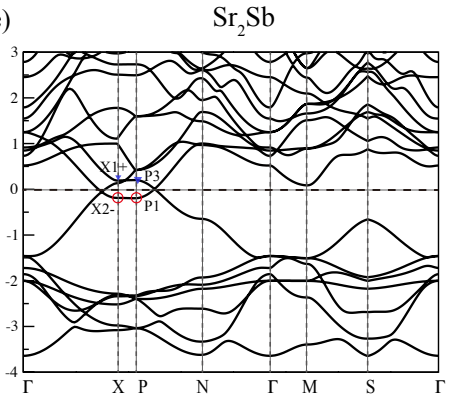

(f)
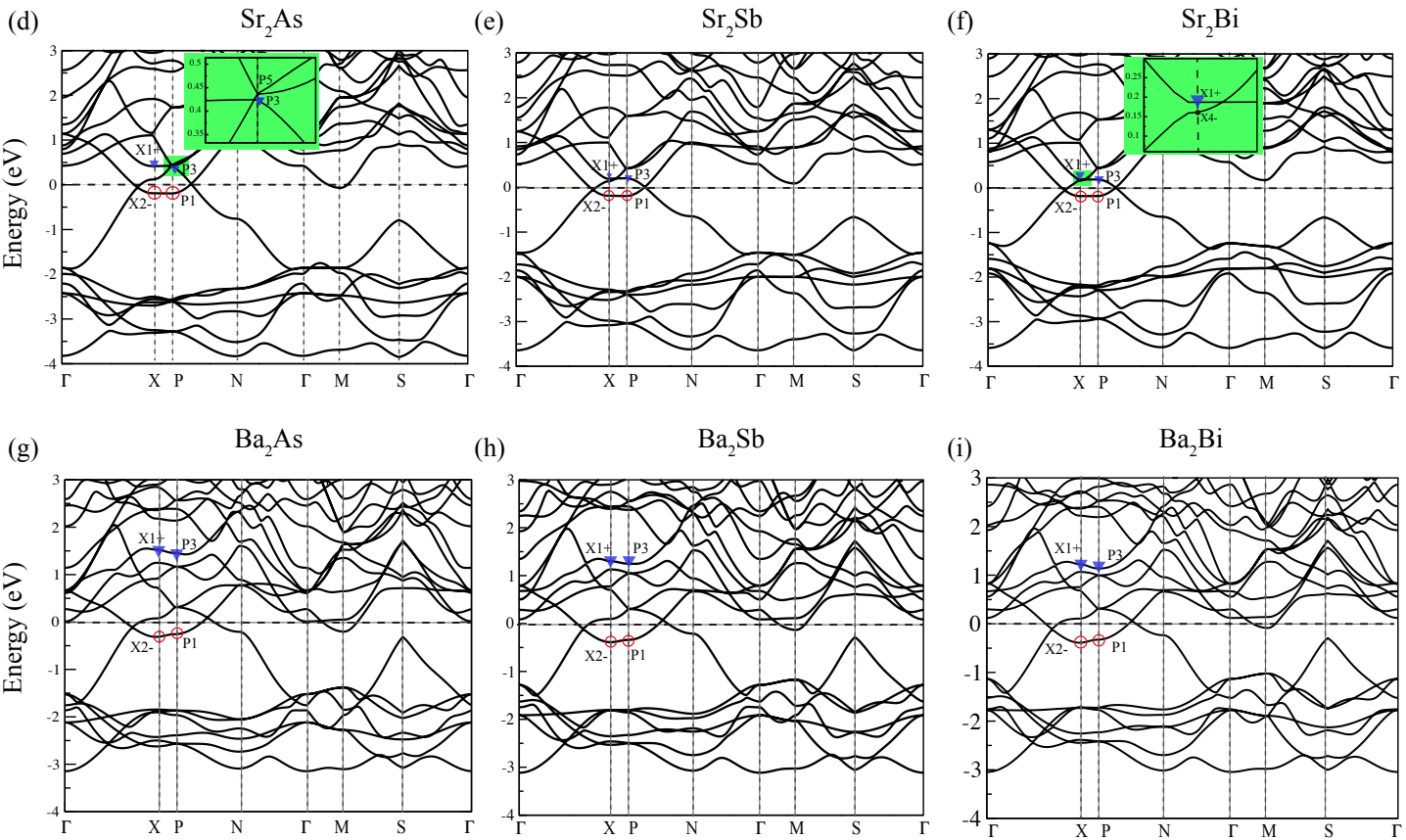

(h)
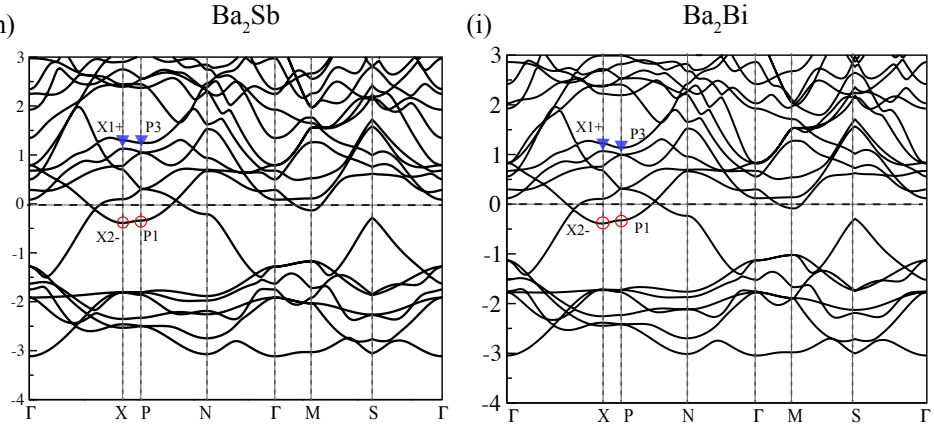

FIG. S2. (Color online) The calculated band structures of $A_{2} B$ family. For the trivial band structures without band inversion, the bands marked by the blue-filled triangles are occupied by charges at vacancies, and the bands marked by red-hollow circles unoccupied. It shows that the band inversion increases as the weights of the pnicogen elements increase.

\section{E. The absorption of hydrogen atoms in $\mathrm{Ba}_{3} \mathrm{CrN}_{3}$}

Table S7 shows the aBRs of $\mathrm{Ca}_{5} \mathrm{P}_{3}$, which are used to understand the absorption of hydrogen atoms in this material. Similar to $\mathrm{Ca}_{5} \mathrm{P}_{3}$, we perform the $\mathrm{BR}$ analysis on $\mathrm{Ba}_{3} \mathrm{CrN}_{3}$ with the space group of $\mathrm{P} 6_{3} / \mathrm{m}$ (\#176), which was previously predicted to be a 1D topological electride with anomalous Dirac plasmon [55]. Fig. S3(a) shows the band structure of $\mathrm{Ba}_{3} \mathrm{CrN}_{3}$, where the obtained irreps are marked out. Based on the aBRs of $\mathrm{Ba}_{3} \mathrm{CrN}_{3}$ shown in Table S8, the BR analysis shows that the blue-solid bands belong to the eBR of $A_{1 g} @ 2 b$, induced by excess electrons at the vacancies $2 b$. By absorbing hydrogen atoms at the vacancies, these bands are removed from $E_{F}$ for $\mathrm{Ba}_{3} \mathrm{CrN}_{3} \mathrm{H}$ [Fig. S3(b)]. The insulating band structure is consistent with recent measurements, since the chargebalanced compound $\mathrm{Ba}_{3} \mathrm{CrN}_{3} \mathrm{H}$ has been obtained in experiments [56]. So the results of hydrogen absorption clearly show that electrides exhibit excess electrons at vacancies.

\section{F. The BR analysis of the predicted electrides}

In order to further understand the eBRs contributed from the states at vacancies, we tabulate the atomic positions and aBRs for $\mathrm{NaBaO}, \mathrm{K}_{2} \mathrm{O}, \mathrm{Sr}_{6} \mathrm{GaN}_{5}$ and $\mathrm{Sr}_{3} \mathrm{P}_{2}$ in Tables S9, S10, S11 and S12, respectively. We here focus on another predicted electride, i.e., $\mathrm{Sr}_{3} \mathrm{P}_{2}$. The generalized gradient approximation band structure of $\mathrm{Sr}_{3} \mathrm{P}_{2}$ with the space group of İ̄2d (\#122) is presented in Fig. S3(c). The BR analysis indicates that two blue-colored bands (above 

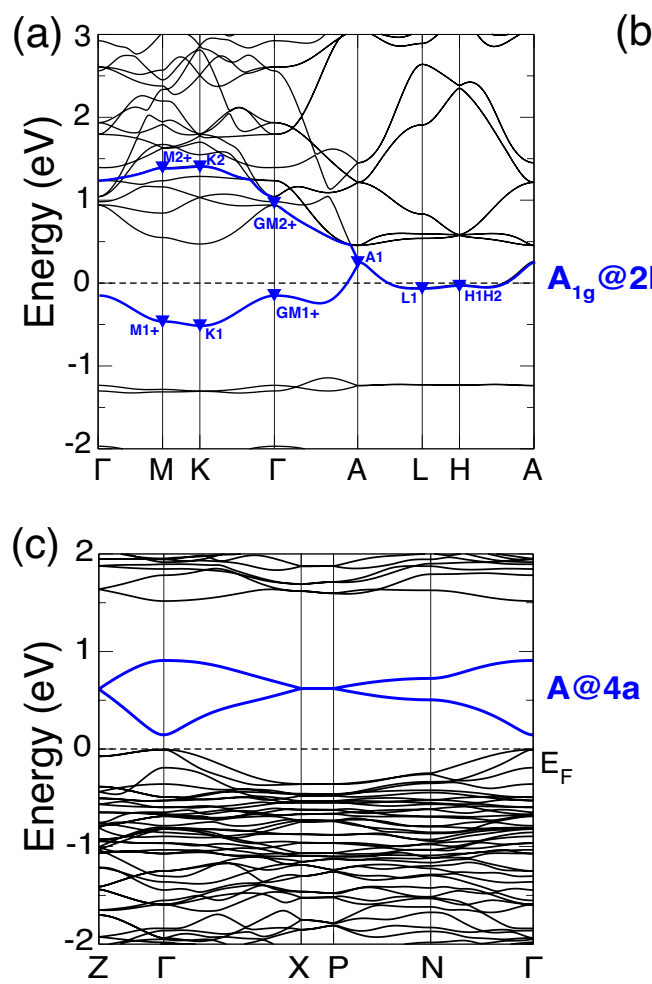

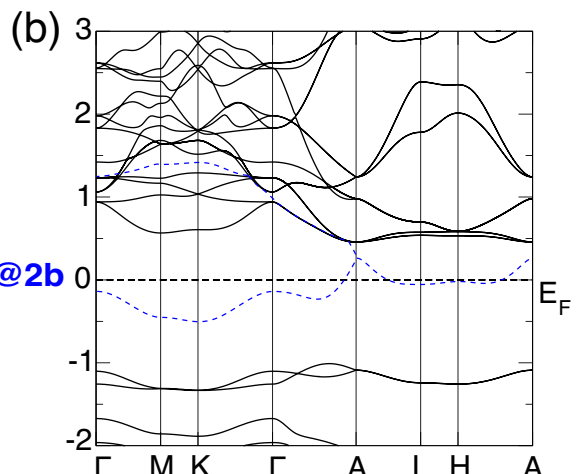

(d)

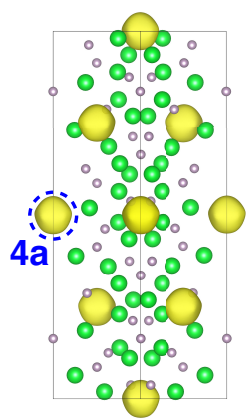

FIG. S3. (Color online) The band structures of $\mathrm{Ba}_{3} \mathrm{CrN}_{3}$ (a), $\mathrm{Ba}_{3} \mathrm{CrN}_{3} \mathrm{H}$ (b) and $\mathrm{Sr}_{3} \mathrm{P}_{2}$ (c). The floating bands (blue-solid) denote electronic states of excess electrons, whose eBRs can be well determined by the obtained irreps. To facilitate the comparison, the irreps of particular bands are marked out, and the "disappeared" floating bands are depicted by blue-dashed lines in (b) for $\mathrm{Ba}_{3} \mathrm{CrN}_{3} \mathrm{H}$. (d) The PED of $\mathrm{Sr}_{3} \mathrm{P}_{2}$.

TABLE S7. Atomic positions and aBRs of $\mathrm{Ca}_{5} \mathrm{P}_{3}$ (space group $P 6_{3} / \mathrm{mcm}$ ).

\begin{tabular}{c|c|c|c|cc|c}
\hline \hline Atom & WKS $(q)$ & Site symm. & Conf. & Irreps $(\rho)$ & aBRs $(\rho @ q)$ \\
\hline $\mathrm{Ca}(1)$ & $4 d$ & 32 & $3 p^{6} 4 s^{2}$ & $s$ & $: A_{1}$ & $A_{1} @ 4 d$ \\
\cline { 4 - 6 } & & & & $p_{z}$ & $: A_{2}$ & $A_{2} @ 4 d$ \\
& & & & $p_{x}, p_{y}$ & $: E$ & $E @ 4 d$ \\
\hline $\mathrm{Ca}(2)$ & $6 g$ & $m m 2$ & $3 p^{6} 4 s^{2}$ & $s$ & $: A_{1}$ & $A_{1} @ 6 g$ \\
\cline { 4 - 6 } & & & $p_{z}$ & $: A_{1}$ & $A_{1} @ 6 g$ \\
& & & $p_{y}$ & $: B_{2}$ & $B_{2} @ 6 g$ \\
& & & & $p_{x}$ & $: B_{1}$ & $B_{1} @ 6 g$ \\
\hline $\mathrm{P}$ & $6 g$ & $m m 2$ & $3 s^{2} 3 p^{3}$ & $s$ & $: A_{1}$ & $A_{1} @ 6 g$ \\
& & & $p_{z}$ & $: A_{1}$ & $A_{1} @ 6 g$ \\
& & & $p_{y}$ & $: B_{2}$ & $B_{2} @ 6 g$ \\
& & & & $p_{x}$ & $: B_{1}$ & $B_{1} @ 6 g$ \\
\hline \hline
\end{tabular}

$\left.E_{F}\right)$ form the eBR of $A @ 4 b$. The PED of the two bands is plotted in Fig. S3(d), agreeing well with the BR analysis. Since the conduction bands of both $\mathrm{Sr}_{3} \mathrm{P}_{2}$ and $\mathrm{Sr}_{6} \mathrm{GaN}_{5}$ are well separated from other bands, and the charge centers of these conduction bands are well defined at the vacancies, we propose that $\mathrm{Sr}_{3} \mathrm{P}_{2}$ and $\mathrm{Sr}_{6} \mathrm{GaN}_{5}$ are potential electrides; namely, they could be electrides after electron doping. As shown in Figs. 5(a,c) in the main text, the floating bands of $\mathrm{K}_{2} \mathrm{O}$ and $\mathrm{Sr}_{6} \mathrm{GaN}_{5}$ are the highest conduction bands. To occupy these bands, electron doping with suitable elements is employed. For $\mathrm{K}_{2} \mathrm{O}$ and $\mathrm{Sr}_{6} \mathrm{GaN}_{5}, 50 \%$ oxygen and $50 \%$ gallium are doped with $\mathrm{F}$ and Ge, respectively. The electronic structures of $\mathrm{K}_{2} \mathrm{O}_{0.5} \mathrm{~F}_{0.5}$ and $\mathrm{Sr}_{6} \mathrm{Ga}_{0.5} \mathrm{Ge}_{0.5} \mathrm{~N}_{5}$ are studied with the virtual crystal approximation method, and the results are shown in Fig. S4. It is clearly that the floating bands are half filled, and the overall band structures almost don't change except an overall band structure shift. 
TABLE S8. Atomic positions and aBRs of $\mathrm{Ba}_{3} \mathrm{CrN}_{3}$ (space group $P 6_{3} / m$ ).

\begin{tabular}{|c|c|c|c|c|c|c|}
\hline$\overline{\text { Atom }}$ & WKS $(q)$ & Site symm. & Conf. & \multicolumn{2}{|c|}{$\operatorname{Irreps}(\rho)$} & $\overline{\operatorname{aBRs}(\rho @ q)}$ \\
\hline \multirow[t]{2}{*}{$\mathrm{Ba}$} & \multirow[t]{2}{*}{$6 h$} & \multirow[t]{2}{*}{$m$} & \multirow[t]{2}{*}{$5 s^{2} 6 s^{2} 5 p^{6}$} & $s$ & $: A^{\prime}$ & $A^{\prime} @ 6 h$ \\
\hline & & & & $\begin{array}{c}p_{x}, p_{y} \\
p_{z}\end{array}$ & $\begin{array}{l}: A^{\prime} \\
: A^{\prime \prime}\end{array}$ & $\begin{array}{l}A^{\prime} @ 6 h \\
A^{\prime \prime} @ 6 h\end{array}$ \\
\hline \multirow[t]{3}{*}{$\mathrm{Cr}$} & \multirow[t]{3}{*}{$2 c$} & \multirow[t]{3}{*}{$\overline{6}$} & \multirow[t]{3}{*}{$3 d^{5} 4 s^{1}$} & $s$ & $: A^{\prime}$ & $A^{\prime} @ 2 c$ \\
\hline & & & & $\begin{array}{l}p_{z} \\
p_{x} \\
p_{y}\end{array}$ & $\begin{array}{l}: A^{\prime \prime} \\
:{ }^{2} E^{\prime} \\
:{ }^{1} E^{\prime}\end{array}$ & $\begin{array}{l}A^{\prime \prime} @ 2 c \\
{ }^{2} E^{\prime} @ 2 c \\
{ }^{1} E^{\prime} @ 2 c\end{array}$ \\
\hline & & & & $\begin{array}{c}d_{z^{2}} \\
d_{x^{2}-y^{2}} \\
d_{x z} \\
d_{x y} \\
d_{y z}\end{array}$ & $\begin{array}{l}: A^{\prime} \\
:{ }^{2} E^{\prime} \\
:{ }^{2} E^{\prime \prime} \\
:{ }^{1} E^{\prime} \\
:{ }^{1} E^{\prime \prime}\end{array}$ & $\begin{array}{c}A^{\prime} @ 2 c \\
{ }^{2} E^{\prime} @ 2 c \\
{ }^{2} E^{\prime \prime} @ 2 c \\
{ }^{1} E^{\prime} @ 2 c \\
{ }^{1} E^{\prime \prime} @ 2 c\end{array}$ \\
\hline \multirow[t]{2}{*}{$\mathrm{N}$} & \multirow[t]{2}{*}{$6 h$} & \multirow[t]{2}{*}{$m$} & \multirow[t]{2}{*}{$2 s^{2} 2 p^{3}$} & $s$ & $: A^{\prime}$ & $A^{\prime} @ 6 h$ \\
\hline & & & & $\begin{array}{c}p_{x}, p_{y} \\
p_{z}\end{array}$ & $\begin{array}{l}: A^{\prime} \\
: A^{\prime \prime}\end{array}$ & $\begin{array}{l}A^{\prime} @ 6 h \\
A^{\prime \prime} @ 6 h \\
\end{array}$ \\
\hline
\end{tabular}

TABLE S9. Atomic positions and aBRs of $\mathrm{NaBaO}$ (space group $P 4 / \mathrm{nmm}$ ).

\begin{tabular}{c|c|c|c|cc|c}
\hline \hline Atom & WKS $(q)$ & Site symm. & Conf. & \multicolumn{2}{|c|}{ Irreps $(\rho)$} & aBRs $(\rho @ q)$ \\
\hline $\mathrm{Ba}$ & $2 c$ & $4 m m$ & $5 s^{2} 6 s^{2} 5 p^{6}$ & $s$ & $: A_{1}$ & $A_{1} @ 2 c$ \\
\cline { 4 - 6 } & & & & $p_{z}$ & $: A_{1}$ & $A_{1} @ 2 c$ \\
& & & & $p_{x}, p_{y}$ & $: E$ & $E @ 2 c$ \\
\hline $\mathrm{O}$ & $2 c$ & $4 m m$ & $2 s^{2} 2 p^{4}$ & $s$ & $: A_{1}$ & $A_{1} @ 2 c$ \\
\cline { 4 - 7 } & & & & $p_{z}$ & $: A_{1}$ & $A_{1} @ 2 c$ \\
& & & & $p_{x}, p_{y}$ & $: E$ & $E @ 2 c$ \\
\hline $\mathrm{Na}$ & $2 c$ & $4 m m$ & $3 s^{1}$ & $s$ & $: A_{1}$ & $A_{1} @ 2 c$ \\
\hline \hline
\end{tabular}

TABLE S10. Atomic positions and aBRs of $\mathrm{K}_{2} \mathrm{O}$ (space group $F m \overline{3} m$ ).

\begin{tabular}{c|c|c|c|cc|c}
\hline \hline Atom & WKS $(q)$ & Site symm. & Conf. & \multicolumn{2}{|c|}{ Irreps $(\rho)$} & aBRs $(\rho @ q)$ \\
\hline $\mathrm{O}$ & $4 a$ & $m \overline{3} m$ & $3 p^{6} 4 s^{2}$ & $s$ & $: A_{1 g}$ & $A_{1 g} @ 4 a$ \\
\cline { 4 - 5 } & & & & $p_{x}, p_{y}, p_{z}$ & $: T_{1 u}$ & $T_{1 u} @ 4 a$ \\
\hline $\mathrm{K}$ & $8 c$ & $\overline{4} 3 m$ & $3 p^{6} 4 s^{2}$ & $s$ & $: A_{1}$ & $A_{1} @ 8 c$ \\
\cline { 4 - 6 } & & & & $p_{x}, p_{y}, p_{z}$ & $: T_{1}$ & $T_{1} @ 8 c$ \\
\hline \hline
\end{tabular}

TABLE S11. Atomic positions and aBRs of $\mathrm{Sr}_{6} \mathrm{GaN}_{5}$ (space group $P 6_{3} / \mathrm{mcm}$ ).

\begin{tabular}{|c|c|c|c|c|c|c|}
\hline Atom & WKS $(q)$ & Site symm. & Conf. & \multicolumn{2}{|c|}{$\operatorname{Irreps}(\rho)$} & $\operatorname{aBRs}(\rho @ q)$ \\
\hline \multirow[t]{3}{*}{$\mathrm{Sr}$} & \multirow[t]{3}{*}{$12 k$} & \multirow[t]{3}{*}{$m$} & \multirow[t]{3}{*}{$5 s^{2} 6 s^{2} 5 p^{6}$} & $s$ & $: A^{\prime}$ & $A^{\prime} @ 12 k$ \\
\hline & & & & $p_{x} / p_{y}$ & $: A^{\prime}$ & $A^{\prime} @ 12 k$ \\
\hline & & & & $p_{z}$ & $: A^{\prime \prime}$ & $A^{\prime \prime} @ 12 k$ \\
\hline \multirow[t]{6}{*}{$\mathrm{Ga}$} & \multirow[t]{6}{*}{$2 a$} & \multirow[t]{6}{*}{$\overline{6} 2 m$} & \multirow[t]{6}{*}{$4 s^{2} 4 p^{1}$} & $s$ & $: A_{1}^{\prime}$ & $A_{1}^{\prime} @ 2 a$ \\
\hline & & & & $p_{z}$ & $: A_{2}^{\prime \prime}$ & $A_{2}^{\prime \prime} @ 2 a$ \\
\hline & & & & $p_{y}$ & $: E^{\prime}$ & $E^{\prime} @ 2 a$ \\
\hline & & & & $d_{z^{2}}$ & $: A_{1}^{\prime}$ & $A_{1}^{\prime} @ 2 a$ \\
\hline & & & & $d_{x^{2}-y^{2}}, d_{x y}$ & $: E^{\prime}$ & $E^{\prime} @ 2 a$ \\
\hline & & & & $d_{x z}, d_{y z}$ & $: E^{\prime \prime}$ & $E^{\prime \prime} @ 2 a$ \\
\hline \multirow[t]{3}{*}{$\mathrm{N}(1)$} & \multirow[t]{3}{*}{$4 d$} & \multirow[t]{3}{*}{32} & \multirow[t]{3}{*}{$2 s^{2} 2 p^{3}$} & $s$ & $: A_{1}$ & $A_{1} @ 4 d$ \\
\hline & & & & $p_{z}$ & $: A_{2}$ & $A_{2} @ 4 d$ \\
\hline & & & & $p_{x}, p_{y}$ & $: E$ & $E @ 4 d$ \\
\hline \multirow[t]{4}{*}{$\mathrm{N}(2)$} & \multirow[t]{4}{*}{$6 g$} & \multirow[t]{4}{*}{$m m 2$} & \multirow[t]{4}{*}{$2 s^{2} 2 p^{3}$} & $s$ & $: A_{1}$ & $A_{1} @ 6 g$ \\
\hline & & & & $p_{z}$ & $: A_{1}$ & $A_{1} @ 6 g$ \\
\hline & & & & $p_{y}$ & $: B_{2}$ & $B_{2} @ 6 g$ \\
\hline & & & & $p_{x}$ & $: B_{1}$ & $B_{1} @ 6 g$ \\
\hline
\end{tabular}




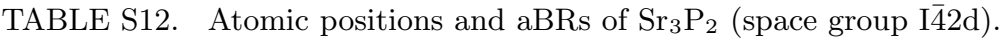

\begin{tabular}{|c|c|c|c|c|c|}
\hline \begin{tabular}{l|l|} 
Atom \\
\end{tabular} & $\overline{\mathrm{WKS}(q)}$ & Site symm & Conf. & Irreps $(\rho)$ & $\overline{\operatorname{aBRs}(\rho @ q)}$ \\
\hline $\operatorname{Sr}(1)$ & $16 e$ & 1 & $5 s^{2} 6 s^{2} 5 p^{6}$ & $s / p_{x} / p_{y} / p_{z}: A$ & $A @ 16 e$ \\
\hline $\operatorname{Sr}(2)$ & $16 e$ & 1 & $5 s^{2} 6 s^{2} 5 p^{6}$ & $s / p_{x} / p_{y} / p_{z}: A$ & $A @ 16 e$ \\
\hline $\operatorname{Sr}(3)$ & $16 e$ & 1 & $5 s^{2} 6 s^{2} 5 p^{6}$ & $s / p_{x} / p_{y} / p_{z}: A$ & $A @ 16 e$ \\
\hline \multirow[t]{2}{*}{$\mathrm{P}(1)$} & \multirow[t]{2}{*}{$8 c$} & \multirow[t]{2}{*}{2} & \multirow[t]{2}{*}{$3 s^{2} 3 p^{3}$} & $s / p_{z}$ & $A @ 8 c$ \\
\hline & & & & $p_{x} / p_{y}$ & $B @ 8 c$ \\
\hline \multirow[t]{2}{*}{$\mathrm{P}(2)$} & \multirow[t]{2}{*}{$8 d$} & \multirow[t]{2}{*}{2} & \multirow[t]{2}{*}{$3 s^{2} 3 p^{3}$} & $s / p_{z}$ & $A @ 8 d$ \\
\hline & & & & $p_{x} / p_{y}$ & $B @ 8 d$ \\
\hline $\mathrm{P}(3)$ & $16 e$ & 1 & $3 s^{2} 3 p^{3}$ & $s / p_{x} / p_{y} / p_{z}: A$ & $A @ 16 e$ \\
\hline
\end{tabular}
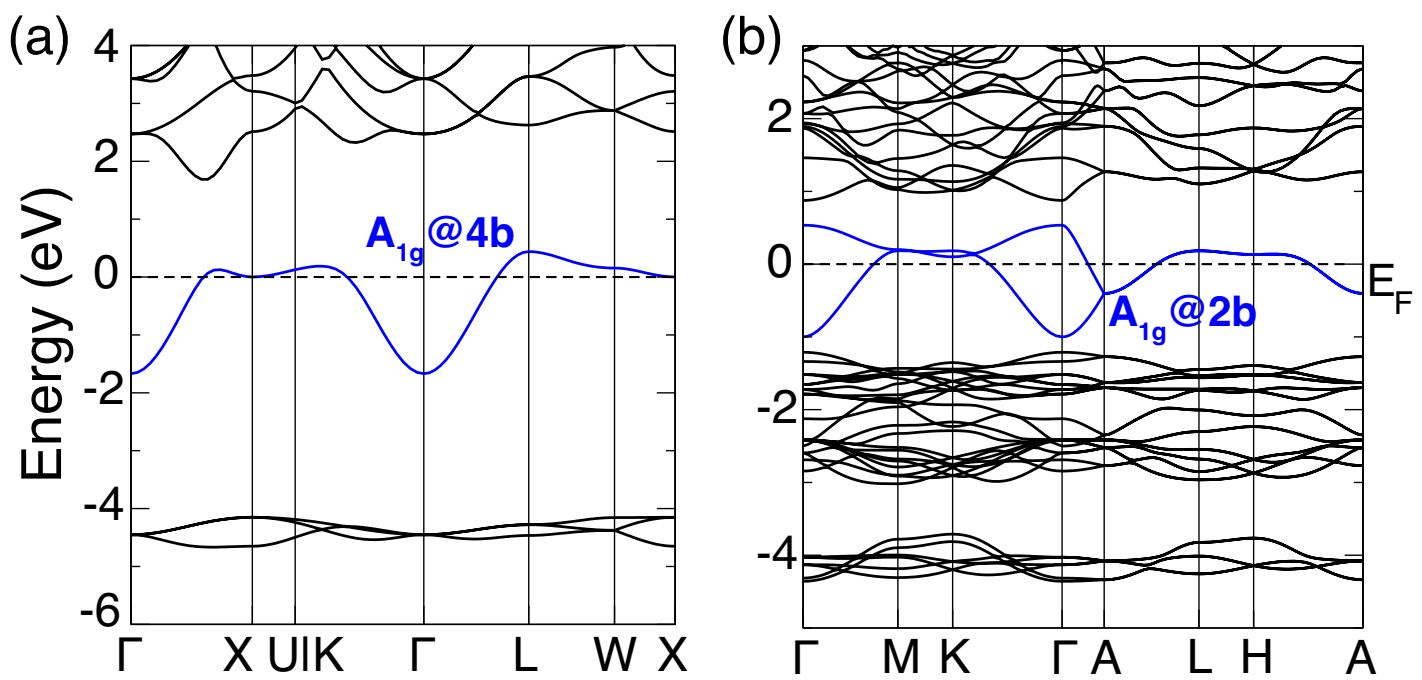

FIG. S4. (Color online) (a,b) The band structures of $\mathrm{K}_{2} \mathrm{O}_{0.5} \mathrm{~F}_{0.5}$ and $\mathrm{Sr}_{6} \mathrm{Ga}_{0.5} \mathrm{Ge}_{0.5} \mathrm{~N}_{5}$, respectively. 